\title{
Cohomological equations and invariant distributions for minimal circle diffeomorphisms
}

\author{
Artur Avila and Alejandro Kocsard
}

March 24, 2018

\begin{abstract}
Given any smooth circle diffeomorphism with irrational rotation number, we show that its invariant probability measure is the only invariant distribution (up to multiplication by a real constant). As a consequence of this, we show that the space of real $C^{\infty}$-coboundaries of such a diffeomorphism is closed in $C^{\infty}(\mathbb{T})$ if and only if its rotation number is Diophantine.
\end{abstract}

\section{Introduction}

Cohomological equations appear very frequently in different contexts in dynamical systems. In fact, many problems, specially those concerning with certain forms of rigidity and stability, can be reduced to analyze the existence of solutions (in certain regularity classes) of some cohomological equations (see [Kat01, Kat03, for general reference, Ghy89, Hur02 for applications to the study of foliations and Ghy01, Nav02] for cohomological aspects of group actions on the circle).

In the case the dynamics is given by a diffeomorphism $f$ on a manifold $M$, the most basic cohomological equation (and the only kind we shall consider from now on) is a first order linear difference equation

$$
u f-u=\phi,
$$

where $\phi: M \rightarrow \mathbb{R}$ is given and $u: M \rightarrow \mathbb{R}$ is the unknown of the problem.

In this work we shall mainly concern with cohomological equations in the smooth category. In fact, most of the time we will assume that the data of the equations, i.e. the diffeomorphism $f$ and the function $\phi$ in (1.1), are $C^{\infty}$ and be interested in the existence of smooth solutions.

By analogy with the cohomology of groups, we can consider the function $\phi$ in (1.1) as being a smooth cocycle over $f$, and we say that $\phi$ is a (smooth) coboundary whenever 
(1.1) admits a $C^{\infty}$ solution. Of course, this leads us to define the first cohomology space $H^{1}\left(f, C^{\infty}(M)\right)$ (see $\$ 2.2$ for details).

In general these cohomology spaces could be rather "wild" (e.g. its natural topology is non-Hausdorff), and so it is rather hard to study the structure of these spaces. However, we can distinguish two aspects that appear as the fundamental characters in the analysis of $H^{1}\left(f, C^{\infty}(M)\right)$ :

(i) the first one is the space of $f$-invariant distributions in the sense of Schwartz (see $\$ 2.2$ for details);

(ii) and the second one is the concept of cohomological stability (see Definition 2.1).

It is important to remark that, in general, the second problem is considerably much harder than the first one. The work of Heafliger and Banghe [HB83] is a good testimony of this.

\subsection{Cohomological equations over quasi-periodic systems}

Equations like (1.1) where $f=R_{\alpha}: \mathbb{T}^{d} \rightarrow \mathbb{T}^{d}$ is an ergodic rigid rotation on the $d$-torus appear as "linearized equations" in many KAM problems. In such a case we have a very clear and simple description of the smooth cohomology: it can be shown the Haar measure on $\mathbb{T}^{d}$ is the only (modulo multiplication by a constant) $R_{\alpha}$-invariant distribution, and $R_{\alpha}$ is cohomologically $C^{\infty}$-stable if and only if $\alpha$ is a Diophantine vector (see $\$ 2.3$ for definitions).

Nevertheless, the general situation is much more complicated: when $f$ is an arbitrary quasi-periodic diffeomorphism, i.e. $f \in \operatorname{Diff}^{\infty}\left(\mathbb{T}^{d}\right)$ is topologically conjugate to an ergodic rigid rotation, in general it is very hard to determine the space of $f$-invariant distributions and the cohomological stability issue seems to be even subtler.

For instance, the problem of computing the $C^{\infty}$ first cohomology space of an arbitrary minimal circle diffeomorphism has been included in several compilations of open problems concerning group actions and foliations (see Lan94, Ghy08 for instance).

In this article we solve this problem proving the following

Theorem A. Let $F: \mathbb{T} \rightarrow \mathbb{T}$ be an orientation-preserving $C^{\infty}$ diffeomorphism with irrational rotation number and $\mu$ be its only invariant probability measure. Then, up to multiplication by a real constant, $\mu$ is the only F-invariant distribution.

Yoccoz, in his $\mathrm{PhD}$ thesis, showed that within the set of smooth circle diffeomorphisms with fixed rotation number $\alpha \in(\mathbb{R} \backslash \mathbb{Q}) / \mathbb{Z}$ those which are smoothly conjugate to the rotation $R_{\alpha}: x \mapsto x+\alpha$ form a dense subset (see Chapter III in Yoc95]). To some extent, our Theorem A can be considered as a "cocycle version" of his result.

It is important to remark that Theorem A is absolutely one-dimensional and cannot be extended to higher dimensions. In fact, in a forthcoming article AK09 we will show the existence of smooth diffeomorphisms of $\mathbb{T}^{2}$ which are topologically conjugate to rigid rotations and exhibit higher order invariant distributions.

On the other hand, as an almost straightforward consequence of Theorem A, we can obtain the following 
Corolary B. A minimal $C^{\infty}$ circle diffeomorphism is cohomologically $C^{\infty}$-stable if and only if its rotation number is Diophantine.

\subsection{Denjoy-Koksma inequality improved}

Given a circle homeomorphism $F$ with irrational rotation number $\rho(F)$ and a real function $\phi: \mathbb{T} \rightarrow \mathbb{R}$, the classical Denjoy-Koksma inequality affirms that the Birkhoff sums satisfy

$$
\left|\sum_{i=1}^{q_{n}-1} \phi\left(f^{i}(x)\right)-q_{n} \int_{\mathbb{T}} \phi \mathrm{d} \mu\right| \leq \operatorname{Var}(\phi), \quad \forall x \in \mathbb{T},
$$

whenever $\phi$ has bounded variation, $\mu$ is the only $F$-invariant probability measure and $q_{n}$ is a denominator of a rational approximation of $\rho(F)$ given by the continued fraction algorithm (see $\S 2.3 .2$ and Proposition 4.2 for details).

Nevertheless, when $F$ is a $C^{3}$ diffeomorphism and $\phi$ is the log-derivative cocycle, i.e. $\phi=\log D F$, Herman showed (see Corollary 2.5.2 of Chapter VII in Her79]) that the previous estimate can be improved. In fact, he proved that $\log D F^{q_{n}}=\sum_{i=0}^{q_{n}-1} \log D F \circ$ $F^{i}$ converges uniformly to zero, as $n \rightarrow \infty$. The interested reader can also find a hard version of this result in Yoc95.

Here, as a consequence of Theorem 7.1, which is nothing but a finite regularity version of Theorem A, we get the following result which can be considered as a generalization of Herman result for arbitrary cocycles:

Corollary C. If $F$ is $C^{11}$ and $\phi$ is $C^{1}$, it holds

$$
\left\|\sum_{i=0}^{q_{n}-1} \phi \circ F^{i}-q_{n} \int_{\mathbb{T}} \phi \mathrm{d} \mu\right\|_{C^{0}} \rightarrow 0, \quad \text { as } n \rightarrow \infty .
$$

\subsection{Some open questions}

At this point it seems to be natural to analyze the first cohomological space of higher dimension quasi-periodic diffeomorphisms.

As we have already mentioned above, in a forthcoming article [AK09] we will show there exist quasi-periodic diffeomorphisms on higher dimensional tori exhibiting higher order invariant distributions.

However, all the examples we know so far have Liouville rotation vectors and are cohomologically $C^{\infty}$-unstable. So it is reasonable to propose the following

Question 1.1. Let $\alpha \in \mathbb{R}^{d}$ be an irrational vector and $f \in \operatorname{Diff}_{+}^{\infty}\left(\mathbb{T}^{d}\right)$ be topologically conjugate to the rigid rotation $R_{\alpha}: x \mapsto x+\alpha$.

Is it true that $f$ is cohomologically $C^{\infty}$-stable if an only if $\alpha$ is Diophantine?

Question 1.2. Let $\alpha$ and $f$ as above. If $\alpha$ is Diophantine, then does it hold

$$
\operatorname{dim} \mathcal{D}^{\prime}(f)=1 ?
$$

It is interesting to remark that Question 1.2 could be a first step toward an eventually higher dimensional version of Herman-Yoccoz linearization theorem. 


\section{Acknowledgments}

We are very grateful to Giovanni Forni for several useful discussions and for having pointed out a (serious) mistake in the very first proof of Theorem 6.1.

A.K. would also like to thank Étienne Ghys for having brought the problem that motivated Theorem A to his attention, and Anatole Katok for insightful conversations.

We thank the anonymous referee for his/her valuable suggestions that helped to improve the exposition of the paper.

This research was partially conducted during the period A.A. served as a Clay Research Fellow. A.K. was partially supported by FAPERJ-Brazil.

\section{Preliminaries}

\subsection{General notations}

Along this article $M$ will denote an arbitrary smooth boundaryless manifold. Given $r \in \mathbb{N}_{0} \cup\{\infty\}$, we write $C^{r}(M)$ for the space of $C^{r}$ real functions on $M$ and $\operatorname{Diff}^{r}(M)$ for the group of $C^{r}$ diffeomorphisms 1 .

Let us recall that when $r$ is finite the uniform $C^{r}$-topology turns $C^{r}(M)$ into a Banach space. On the other hand, we shall consider the space $C^{\infty}(M)$ endowed with its usual Fréchet topology, which can be defined as the projective limit of the family of Banach spaces $\left(C^{r}(M)\right)_{r \in \mathbb{N}}$.

Given any $f \in \operatorname{Diff}^{r}(M), \operatorname{Fix}(f)$ and $\operatorname{Per}(f)$ stand for the set of fixed and periodic points of $f$, respectively. Whenever $M$ is orientable, we write $\operatorname{Diff}_{+}^{r}(M)$ for the subgroup of $C^{r}$ orientation-preserving diffeomorphisms.

The $d$-dimensional torus will be denoted by $\mathbb{T}^{d}$ and will be identified with $\mathbb{R}^{d} / \mathbb{Z}^{d}$. The canonical quotient projection will be denoted by $\pi: \mathbb{R}^{d} \rightarrow \mathbb{T}^{d}$. For simplicity, we shall just write $\mathbb{T}$ for the 1-torus, i.e. the circle.

The symbol Leb $d$ will be used to denote the Lebesgue measure on $\mathbb{R}^{d}$, as well as the Haar probability measure on $\mathbb{T}^{d}$. Once again, for the sake of simplicity, we just write Leb, and also $\mathrm{d} x$, instead of Leb . $_{\text {. }}$.

As usual, we shall identify $C^{r}\left(M, \mathbb{R}^{k}\right)$ with $\left(C^{r}(M)\right)^{k}$, and $C^{r}\left(\mathbb{T}^{d}\right)$ with the space of $\mathbb{Z}^{d}$-periodic real $C^{r}$ functions on $\mathbb{R}^{d}$.

In the particular case of $C^{r}$ real functions on $\mathbb{T}$, we explicitly define the $C^{r}$-norm on $C^{r}(\mathbb{T})$ (with $\left.0 \leq r<\infty\right)$ by

$$
\|\phi\|_{C^{r}}:=\max _{x \in \mathbb{T}} \max _{0 \leq j \leq r}\left|D^{j} \phi(x)\right|, \quad \forall \phi \in C^{r}(\mathbb{T}) .
$$

Moreover, whenever $I \subset \mathbb{R}$ is a compact interval and $\psi \in C^{r}(\mathbb{R})$, we define

$$
\left\|\left.\psi\right|_{I}\right\|_{C^{r}}:=\max _{x \in I} \max _{0 \leq j \leq r}\left|D^{j} \psi(x)\right| .
$$

Next, we define the space of lifts of circle diffeomorphisms by

$$
\widetilde{\operatorname{Diff}_{+}^{r}}(\mathbb{T}):=\left\{f \in \operatorname{Diff}_{+}^{r}(\mathbb{R}): f-i d_{\mathbb{R}} \in C^{r}(\mathbb{T})\right\} .
$$

\footnotetext{
${ }^{1}$ As usual, we use the term " $C^{0}$ diffeomorphism" as a synonymous of "homeomorphism".
} 
It can be easily shown that this space is connected and simply connected. In particular, this space can be identified with the universal covering of $\operatorname{Diff}_{+}^{r}(\mathbb{T})$. Making some abuse of notation, we will also denote by $\pi$ the canonical projection $\widetilde{\operatorname{Diff}_{+}^{r}}(\mathbb{T}) \rightarrow \operatorname{Diff}_{+}^{r}(\mathbb{T})$ that associates to each $f \in \widetilde{\operatorname{Diff}_{+}^{r}}(\mathbb{T})$ the only circle diffeomorphism lifted by $f$.

As usual, we write $\rho: \widetilde{\operatorname{Diff}_{+}^{0}}(\mathbb{T}) \rightarrow \mathbb{R}$ for the rotation number function, and we will use the same letter to call the induced map $\rho: \operatorname{Diff}_{+}^{0}(\mathbb{T}) \rightarrow \mathbb{R} / \mathbb{Z}$ (see $\S 1.1$ in $\mathrm{dMvS93}$ for the definitions).

And finally, two important remarks about notation. First, for the sake of simplicity, when dealing with estimates we will use the letter $C$ to denote any positive real constant which may assume different values along the article, even in a single chain of inequalities.

And secondly, we will denote the intervals of the real line regardless of the order of the extremal points, i.e. if $a, b$ are two different points of $\mathbb{R}$, we shall write $(a, b)$ for the only bounded connected component of $\mathbb{R} \backslash\{a, b\}$, independently of the order of the points. Of course, we will follow the same convention for the intervals $[a, b],[a, b)$ and $(a, b]$.

\subsection{Cocycles, coboundaries and invariant distributions}

From now on let us assume our manifold $M$ is closed, i.e. compact and boundaryless, and let $f \in \operatorname{Diff}^{r}(M)$, with $r \in \mathbb{N}_{0} \cup\{\infty\}$. Every $\psi \in C^{k}(M)$, where $0 \leq k \leq r$, can be considered as a $C^{k}$ (real) cocycle over $f$ writing

$$
M \times \mathbb{Z} \ni(x, n) \mapsto \mathcal{S}^{n} \psi(x) \in \mathbb{R},
$$

where $\mathcal{S}^{n} \psi=\mathcal{S}_{f}^{n} \psi$ denotes the Birkhoff sum over $f$ given by

$$
\mathcal{S}^{n} \psi:= \begin{cases}\sum_{i=0}^{n-1} \psi \circ f^{i} & \text { if } n \geq 1 ; \\ 0 & \text { if } n=0 \\ -\sum_{i=1}^{-n} \psi \circ f^{-i} & \text { if } n<0 .\end{cases}
$$

We say that the cocycle $\psi \in C^{k}(M)$ is a $C^{\ell}$ coboundary, with $0 \leq \ell \leq k \leq r$, whenever there exists $u \in C^{\ell}(M)$ solving the following cohomological equation:

$$
u \circ f-u=\psi .
$$

We say that $\phi, \psi \in C^{k}(\mathbb{T})$ are $C^{\ell}$-cohomologous whenever the function $\phi-\psi$ is a $C^{\ell}$ coboundary.

The space of $C^{\ell}$ coboundaries will be denoted by $B\left(f, C^{\ell}(M)\right)$, and since it is clearly a linear subspace of $C^{\ell}(M)$, we can define

$$
H^{1}\left(f, C^{\ell}(M)\right):=C^{\ell}(M) / B\left(f, C^{\ell}(M)\right),
$$

called the first $C^{\ell}$-cohomology space of $f$.

This space $H^{1}\left(f, C^{\ell}(M)\right)$ naturally inherits the quotient topology from $C^{\ell}(M)$. Unfortunately, in general $B\left(f, C^{\ell}(M)\right)$ is not closed in $C^{\ell}(M)$, and therefore, this quotient topology is non-Hausdorff. So, it is reasonable to propose the following 
Definition 2.1. We say that $f$ is cohomologically $C^{\ell}$-stable whenever $B\left(f, C^{\ell}(M)\right)$ is a closed in $C^{\ell}(M)$. On the other hand, we define the first reduced $C^{\ell}$-cohomology space as being

$$
\tilde{H}^{1}\left(f, C^{\ell}(M)\right):=C^{\ell}(M) / \mathrm{cl}_{\ell}\left(B\left(f, C^{\ell}(M)\right)\right),
$$

where $\operatorname{cl}_{\ell}(\cdot)$ denotes the closure in the uniform $C^{\ell}$-topology.

As we have already mentioned in \$1 the study of the structure of the spaces $H^{1}\left(f, C^{\infty}(M)\right)$ and $\tilde{H}^{1}\left(f, C^{\infty}(M)\right)$ naturally leads us to consider the space of $f$ invariant (Schwartz) distributions on $M$.

So, for each $k \in \mathbb{N}_{0}$, let $\mathcal{D}_{k}^{\prime}(M)$ be the topological dual space of $C^{k}(M)$, i.e. the space of distributions of order up to $k$ of $M$. As usual, the dual space of $C^{\infty}(M)$ will be simply denoted by $\mathcal{D}^{\prime}(M)$.

Since all the inclusions $C^{k+1}(M) \hookrightarrow C^{k}(M)$ and $C^{\infty}(M) \hookrightarrow C^{k}(M)$ are continuous and have dense range, we can suppose we have the following chain of inclusions, which are defined modulo unique extensions:

$$
\mathcal{D}_{0}^{\prime}(M) \subset \mathcal{D}_{1}^{\prime}(M) \subset \mathcal{D}_{2}^{\prime}(M) \subset \ldots \subset \mathcal{D}^{\prime}(M) .
$$

Moreover, since we are assuming $M$ is compact, it is well-known that

$$
\mathcal{D}^{\prime}(M)=\bigcup_{k \geq 0} \mathcal{D}_{k}^{\prime}(M) .
$$

On the other hand, any $C^{k}$ diffeomorphism $f$ acts linearly on $C^{k}(M)$ by pull-back, and the adjoint of this action is the linear operator $f_{*}: \mathcal{D}_{k}^{\prime}(M) \rightarrow \mathcal{D}_{k}^{\prime}(M)$ given by

$$
\left\langle f_{*} T, \psi\right\rangle:=\langle T, \psi \circ f\rangle, \quad \forall T \in \mathcal{D}_{k}^{\prime}(M), \forall \psi \in C^{k}(M) .
$$

In this case, $\operatorname{Fix}\left(f_{*}\right)$ is the space of $f$-invariant distributions of order up to $k$ and it will be denoted by $\mathcal{D}_{k}^{\prime}(f)$. Of course, it holds $\mathcal{D}^{\prime}(f)=\bigcup_{k \geq 0} \mathcal{D}_{k}^{\prime}(f)$.

As we mentioned above, there is a tight relation between the space of invariant distributions $\mathcal{D}^{\prime}(f)$ and the reduced cohomology group $\tilde{H}^{1}\left(f, C^{\infty}(M)\right)$. In fact, as a straightforward consequence of Hahn-Banach theorem we get the following

Proposition 2.2. Given any $f \in \operatorname{Diff}^{k}(M)$, with $k \in \mathbb{N}_{0} \cup\{\infty\}$, it holds

$$
\operatorname{cl}_{k}\left(B\left(f, C^{k}(M)\right)\right)=\bigcap_{T \in \mathcal{D}_{k}^{\prime}(f)} \operatorname{ker} T .
$$

In particular, this implies that

$$
\operatorname{dim} \tilde{H}^{1}\left(f, C^{k}(M)\right)=\operatorname{dim} \mathcal{D}_{k}^{\prime}(f) .
$$




\subsection{Arithmetic}

\subsubsection{Diophantine and Liouville vectors}

For any $\boldsymbol{\alpha} \in \mathbb{R}^{d}$ we write

$$
\|\boldsymbol{\alpha}\|:=\operatorname{dist}\left(\boldsymbol{\alpha}, \mathbb{Z}^{d}\right)
$$

Notice that, since $\|\boldsymbol{\alpha}+\mathbf{n}\|=\|\boldsymbol{\alpha}\|$ for every $\mathbf{n} \in \mathbb{Z}^{d}$, we can naturally consider $\|\cdot\|$ as defined on $\mathbb{T}^{d}$

We say $\boldsymbol{\alpha}=\left(\alpha_{1}, \ldots, \alpha_{d}\right) \in \mathbb{R}^{d}$ is irrational if and only if, for any $\left(n_{1}, \ldots, n_{d}\right) \in \mathbb{Z}^{d}$, it holds

$$
\left\|\sum_{i=1}^{d} n_{i} \alpha_{i}\right\|=0 \Longrightarrow n_{i}=0, \text { for } i=1, \ldots, d .
$$

Moreover, the vector $\boldsymbol{\alpha}$ is said to be Diophantine whenever there exist constants $C, \tau>0$ satisfying

$$
\left\|\sum_{i=1}^{d} \alpha_{i} q_{i}\right\| \geq \frac{C}{\max _{i}\left|q_{i}\right|^{\tau}},
$$

for every $\left(q_{1}, \ldots, q_{d}\right) \in \mathbb{Z}^{d} \backslash\{0\}$. On the other hand, an irrational element of $\mathbb{R}^{d}$ which is not Diophantine is called Liouville.

\subsubsection{Continued fractions}

In this paragraph we introduce some common notations and recall some elementary and well-known results about continued fractions (see HW79] for details).

First of all, the Gauss map $A:(0,1) \rightarrow[0,1)$ is defined by

$$
A(x):=\frac{1}{x}-\left\lfloor\frac{1}{x}\right\rfloor .
$$

For each $\alpha \in \mathbb{R} \backslash \mathbb{Q}$ we can associate the sequences $\left(\alpha_{n}\right)_{n \geq 0}$ and $\left(a_{n}\right)_{n \geq 0}$ which are recursively defined by

$$
\begin{gathered}
\alpha_{0}:=\alpha-\lfloor\alpha\rfloor, \quad \alpha_{n}:=A^{n}\left(\alpha_{0}\right), \quad \forall n \geq 1 ; \\
a_{0}:=\lfloor\alpha\rfloor, \quad a_{n+1}:=\left\lfloor\frac{1}{\alpha_{n}}\right\rfloor, \quad \forall n \geq 0 .
\end{gathered}
$$

The $n^{\text {th }}$-convergent of $\alpha$ is defined by

$$
p_{n} / q_{n}:=a_{0}+\frac{1}{a_{1}+\frac{1}{a_{2}+\frac{1}{\cdots+\frac{1}{a_{n}}}}}
$$


and the sequences $\left(p_{n}\right)_{n \geq-2}$ and $\left(q_{n}\right)_{n \geq-2}$ satisfy the following recurrences:

$$
\begin{aligned}
p_{-2}:=0, \quad p_{-1}:=1, \quad p_{n}:=a_{n} p_{n-1}+p_{n-2}, \quad \forall n \geq 0 \\
q_{-2}:=1, \quad q_{-1}:=0, \quad q_{n}:=a_{n} q_{n-1}+q_{n-2}, \quad \forall n \geq 0 .
\end{aligned}
$$

A very important property about $\left(q_{n}\right)$ that we will repeatedly use in the future is the following:

$$
q_{n+1}=\min \left\{q \in \mathbb{N}:\|q \alpha\|<\left\|q_{n} \alpha\right\|\right\}, \quad \forall n \geq 1 .
$$

The reader can easily show that the sequences $\left(p_{n}\right)$ and $\left(q_{n}\right)$ satisfy the following relation:

$$
p_{n-1} q_{n}-p_{n} q_{n-1}=(-1)^{n}, \quad \forall n \geq-1 .
$$

Now let us define the sequence $\left(\beta_{n}\right)_{n \geq-1}$ by

$$
\begin{aligned}
\beta_{-1} & :=1, \\
\beta_{n}: & =\prod_{i=0}^{n} \alpha_{i}, \quad \forall n \geq 0 .
\end{aligned}
$$

By straightforward computations we can show that the sequence $\left(\beta_{n}\right)$ satisfies

$$
\beta_{n}=(-1)^{n}\left(q_{n} \alpha-p_{n}\right)>0
$$

and

$$
\frac{1}{q_{n}+q_{n+1}}<\beta_{n}<\frac{1}{q_{n+1}}
$$

for every $n \geq 0$.

On the other hand, the growth of the sequences $\left(q_{n}\right)$ and $\left(\beta_{n}\right)$ determines whether the number $\alpha$ is Diophantine or Liouville: if $\tau$ denotes any positive real number and we write

$$
\mathcal{L}(\alpha, \tau):=\left\{m \in \mathbb{N}: \beta_{m}<\beta_{m-1}^{\tau}\right\},
$$

it is very easy to verify that $\alpha$ is Liouville if and only if $\mathcal{L}(\alpha, \tau)$ has infinitely many elements, for every $\tau>1$. In fact, this can be proved rewriting (2.1) for $d=1$ : we have $\alpha$ is Diophantine if and only if there exist constant $C, \tau>0$ such that

$$
\beta_{n}=\left|q_{n} \alpha-p_{n}\right|>\frac{C}{q_{n}^{1+\tau}}, \quad \forall n \geq 0,
$$

and, by estimate (2.11), this is equivalent to

$$
\beta_{n+1}>C \beta_{n}^{1+\tau}, \quad \forall n \geq 0 .
$$




\subsection{Cohomology of minimal rotations on the torus}

Let $\boldsymbol{\alpha}=\left(\alpha_{1}, \ldots, \alpha_{d}\right)$ be an irrational vector in $\mathbb{R}^{d}$. It is well-known that in such a case the rotation $R: \mathbb{T}^{d} \rightarrow \mathbb{T}^{d}$ given by

$$
R: x \mapsto x+\left(\boldsymbol{\alpha}+\mathbb{Z}^{d}\right)
$$

is minimal (i.e. all its orbits are dense in $\mathbb{T}^{d}$ ) and uniquely ergodic, being the Haar measure Leb $_{d}$ the only $R$-invariant Borel probability measure.

Moreover, we have the following result which belongs to the folklore:

Proposition 2.3. Continuing with the notation we introduced above, we have

$$
\mathcal{D}^{\prime}(R)=\mathbb{R} \text { Leb }_{d} \text {. }
$$

On the other hand, $R$ is cohomologically $C^{\infty}$-stable if and only if $\boldsymbol{\alpha}$ is Diophantine.

Proof. Let $\psi \in C^{\infty}\left(\mathbb{T}^{d}\right)$ be such that $\int \psi \mathrm{dLeb}_{d}=0$, and let us consider the Fourier development of $\psi$ :

$$
\psi(x)=\sum_{\mathbf{k} \in \mathbb{Z}^{d} \backslash\{0\}} \hat{\psi}_{k} e^{2 \pi i \mathbf{k} \cdot x} .
$$

Then, the Fourier coefficients of any (integrable) solution of the cohomological equation $\psi=u R-u$ must satisfy the following relation:

$$
\hat{u}_{\mathbf{k}}:=\frac{\hat{\psi}_{\mathbf{k}}}{e^{2 \pi i \mathbf{k} \cdot \boldsymbol{\alpha}}-1}, \quad \forall \mathbf{k} \in \mathbb{Z}^{d} \backslash\{0\} .
$$

If $\left\{U_{n}\right\}_{n \geq 1}$ is any sequence of finite subsets of $\mathbb{Z}^{d}$ such that $\bigcup_{n>1} U_{n}=\mathbb{Z}^{d} \backslash\{0\}$ and $U_{n} \subset U_{n+1}$, for every $n \geq 1$, then one can define the trigonometric polynomials

$$
\begin{aligned}
\psi_{n}(x) & :=\sum_{\mathbf{k} \in U_{n}} \hat{\psi}_{\mathbf{k}} e^{2 \pi i \mathbf{k} \cdot x} ; \\
u_{n}(x) & :=\sum_{\mathbf{k} \in U_{n}} \hat{u}_{\mathbf{k}} e^{2 \pi i \mathbf{k} \cdot x}, \quad \text { for } n \geq 1 .
\end{aligned}
$$

Since $u_{n} R-u_{n}=\psi_{n}$, we have $\psi_{n} \in B\left(R, C^{\infty}\left(\mathbb{T}^{d}\right)\right)$, and clearly $\psi_{n} \rightarrow \psi$ in the $C^{\infty}$-topology. Thus, $\psi \in \operatorname{cl}_{\infty}\left(B\left(f, C^{\infty}\left(\mathbb{T}^{d}\right)\right)\right)$. By Proposition 2.2 , we conclude that $\mathcal{D}^{\prime}(R)=$ RLeb $_{d}$.

Now, when $\boldsymbol{\alpha}$ is Diophantine it is easy to verify that the Fourier coefficients $\left(\hat{u}_{\mathbf{k}}\right)_{\mathbf{k} \in \mathbb{Z}^{d}}$ decay sufficiently fast at infinity to guarantee that

$$
u(x):=\sum_{\mathbf{k} \in \mathbb{Z}^{d} \backslash\{0\}} \hat{u}_{\mathbf{k}} e^{2 \pi i \mathbf{k} \cdot x},
$$

defines a $C^{\infty}$ function which turns to be a solution for the cohomological equation $u R-u=\psi$. Applying Proposition 2.2 once again, we obtain

$$
B\left(f, C^{\infty}\left(\mathbb{T}^{d}\right)\right)=\operatorname{ker} \operatorname{Leb}_{d}=\operatorname{cl}_{\infty}\left(B\left(f, C^{\infty}\left(\mathbb{T}^{d}\right)\right)\right),
$$


and therefore, $R$ is cohomologically $C^{\infty}$-stable.

On the other hand, when $\boldsymbol{\alpha}$ is Liouville it is possible to find a sequence $\left(\mathbf{n}_{j}\right)_{j \geq 1} \subset$ $\mathbb{Z}^{d} \backslash\{0\}$ satisfying $\mathbf{n}_{j} \rightarrow \infty$, as $j \rightarrow+\infty$ and

$$
\left\|\sum_{i=1}^{d} \alpha_{i} n_{j, i}\right\| \leq \frac{1}{\max _{i}\left|n_{j, i}\right|^{j}}, \quad \forall j \geq 1,
$$

where, of course, $\mathbf{n}_{j}=\left(n_{j, 1}, \ldots, n_{j, d}\right)$.

This clearly implies that writing

$$
\psi(x):=\sum_{j \in \mathbb{N}}\left[\left(e^{2 \pi i \mathbf{n}_{j} \cdot \alpha}-1\right) e^{2 \pi i \mathbf{n}_{j} \cdot x}+\left(e^{-2 \pi i \mathbf{n}_{j} \cdot \alpha}-1\right) e^{-2 \pi i \mathbf{n}_{j} \cdot x}\right]
$$

we get $\psi \in C^{\infty}\left(\mathbb{T}^{d}\right) \cap$ ker $\operatorname{Leb}_{d}$. However, $\psi \notin B\left(R, C^{\infty}\left(\mathbb{T}^{d}\right)\right)$ because the Fourier coefficients $\left(\hat{u}_{\mathbf{k}}\right)$ of an eventual solution of the cohomological equation given by (2.15) satisfy

$$
\hat{u}_{\mathbf{n}_{j}}=1, \quad \forall j \in \mathbb{N},
$$

with $\mathbf{n}_{j} \rightarrow \infty$, as $j \rightarrow \infty$.

\section{Proof of the corollaries}

Since the proof of Theorem A is rather technical, we will start proving corollaries B and $\mathrm{C}$ assuming Theorem $\mathrm{A}$.

\subsection{Corollary B}

Let $F \in \operatorname{Diff}_{+}^{\infty}(\mathbb{T})$ be such that $\rho(F) \in(\mathbb{R} \backslash \mathbb{Q}) / \mathbb{Z}, \mu$ denote the only $F$-invariant probability measure, and $f \in \widetilde{\operatorname{Diff}_{+}^{\infty}}(\mathbb{T})$ be a lift of $F$.

By the unique ergodicity it easily follows that

$$
\int_{\mathbb{T}} \log D f \mathrm{~d} \mu=0 .
$$

On the other hand, by Theorem A we know $\mathcal{D}^{\prime}(F)=\mathbb{R} \mu$. Hence, if we suppose that $F$ is cohomologically $C^{\infty}$-stable, by (3.1) and Proposition 2.2 we conclude $\log D f \in$ $B\left(F, C^{\infty}(\mathbb{T})\right)$, i.e. there exists $u \in C^{\infty}(\mathbb{T})$ satisfying

$$
u F-u=\log D f .
$$

Now, let us write

$$
h^{\prime}:=C^{-1} \exp (-u) \in C^{\infty}(\mathbb{T}),
$$

where $C:=\int_{\mathbb{T}} \exp (-u)$ dLeb. So, in particular $h^{\prime}$ is positive and satisfies $\int_{\mathbb{T}} h^{\prime} \mathrm{dLeb}=1$. Hence, defining

$$
h(x):=\int_{0}^{x} h^{\prime}(t) \mathrm{d} t, \quad \forall x \in \mathbb{R},
$$


we have $h \in \widetilde{\operatorname{Diff}_{+}^{\infty}}(\mathbb{T})$, and by $(3.2)$ we get

$$
D(h \circ f)=(D h \circ f) D f=\left(h^{\prime} \circ f\right) D f=h^{\prime}=D h .
$$

This implies there exists $\rho \in \mathbb{R}$ such that $h f=h+\rho$, and by invariance of the rotation number under conjugacy we have $\rho=\rho(f)$.

Therefore, $F$ is $C^{\infty}$-conjugate to the rigid rotation $x \mapsto x+\rho(F)$, and applying Proposition 2.3 we conclude $\rho(F)$ is Diophantine.

Reciprocally, by Herman-Yoccoz theorem Her79, Yoc84 any circle diffeomorphism with Diophantine rotation number is $C^{\infty}$-conjugate to the rigid rotation, and by Proposition 2.3 it must be cohomologically $C^{\infty}$-stable, as desired.

\subsection{Corollary C}

To prove Corollary $\mathrm{C}$ we will use two different arguments depending on how well we can approximate the rotation number of the diffeomorphism by rational numbers.

When the rotation number is badly approximated, we will use the finite regularity version of Yoccoz linearization theorem (Théorème, page 335 in [Yoc84]). On the other hand, when the rotation number is not "too badly" approximated, we will use a finite regularity version of our Theorem A, that is Theorem 7.1.

So, let $F \in \operatorname{Diff}_{+}^{11}(\mathbb{T})$ be a minimal diffeomorphism, $f \in \widetilde{\operatorname{Diff}_{+}^{11}}(\mathbb{T})$ be a lift of $F, \mu$ be the only $F$-invariant probability measure, and $\phi \in C^{1}(\mathbb{T})$. Let $\left(p_{n}\right)$ and $\left(q_{n}\right)$ be the sequences associated to $\alpha:=\rho(f)$ given by (2.4) and (2.5), respectively, and $\varepsilon>0$ be arbitrary. Then, let us consider the set $\mathcal{L}(\alpha, 11 / 2)$ given by (2.12).

First, let us suppose $\mathcal{L}(\alpha, 11 / 2)$ is finite. Thus, as we have already mentioned at the end of 22.3 .2 in this case $\alpha$ is Diophantine. More precisely, there exists $C>0$ such that

$$
\|q \alpha\| \geq \frac{C}{q^{11 / 2}}, \quad \forall q \in \mathbb{N} .
$$

Then, by Yoccoz linearization theorem Yoc84 we have $F$ is $C^{1}$-conjugate to the rigid rotation $R_{\alpha}$. Therefore, applying Proposition 2.3 we can conclude $\mathcal{D}_{1}^{\prime}(F)=\mathbb{R} \mu$.

On the other hand, if we suppose $\mathcal{L}(\alpha, 11 / 2)$ has infinite elements, we can apply Theorem 7.1 to conclude that $\mathcal{D}_{1}^{\prime}(F)=\mathbb{R} \mu$, too.

In any case, applying Proposition 2.2 we can conclude there exists $u \in C^{1}(\mathbb{T})$ such that

$$
\left\|(u F-u)-\left(\phi-\int_{\mathbb{T}} \phi \mathrm{d} \mu\right)\right\|_{C^{1}} \leq \frac{\varepsilon}{2} .
$$

Writing

$$
\tilde{\phi}:=u F-u+\int_{\mathbb{T}} \phi \mathrm{d} \mu,
$$

it clearly holds

$$
\int_{\mathbb{T}} \tilde{\phi} \mathrm{d} \mu=\int_{\mathbb{T}} \phi \mathrm{d} \mu
$$


Hence, $\|\tilde{\phi}-\phi\|_{C^{1}} \leq \varepsilon / 2$ and

$$
\left(\tilde{\phi}-\int_{\mathbb{T}} \tilde{\phi} \mathrm{d} \mu\right) \in B\left(F, C^{1}(\mathbb{T})\right) .
$$

On the other hand, if we write

$$
M_{n}:=\sup _{x \in \mathbb{R}}\left|f^{q_{n}}(x)-x-p_{n}\right|,
$$

by the minimality of $F$ we get $M_{n} \rightarrow 0$ when $n \rightarrow \infty$, and so, there exists $N \in \mathbb{N}$ such that $\|D u\|_{C^{0}} M_{n} \leq \varepsilon / 2$, provided $n \geq N$.

Finally, applying Denjoy-Koksma inequality (1.2) (see Proposition 4.2 for the precise statement) for $\tilde{\phi}-\phi$ we get

$$
\begin{aligned}
\mid \mathcal{S}^{q_{n}} \phi(x)- & q_{n} \int_{\mathbb{T}} \phi \mathrm{d} \mu \mid \\
& \leq\left|\mathcal{S}^{q_{n}}(\phi-\tilde{\phi})(x)-q_{n} \int_{\mathbb{T}}(\phi-\tilde{\phi}) \mathrm{d} \mu\right|+\left|\mathcal{S}^{q_{n}} \tilde{\phi}(x)-q_{n} \int_{\mathbb{T}} \tilde{\phi} \mathrm{d} \mu\right| \\
& \leq \operatorname{Var}(\phi-\tilde{\phi})+\left|u\left(F^{q_{n}}(x)\right)-u(x)\right| \\
& \leq \int_{\mathbb{T}}|D(\phi-\tilde{\phi})| \mathrm{d} \mu+\|D u\|_{C^{0}} M_{n} \leq\|\phi-\tilde{\phi}\|_{C^{1}}+\frac{\varepsilon}{2} \leq \varepsilon,
\end{aligned}
$$

for every $x \in \mathbb{T}$ and provided $n$ is sufficiently big. Since $\varepsilon$ is arbitrary, Corollary $\mathrm{C}$ is proved.

\section{$4 C^{r}$-estimates for real cocycles}

This section can be considered the starting point of the proof of Theorem A. The principal new result here is Proposition 4.12, which is mainly inspired in the work of Yoccoz Yoc84.

Along this section $F$ will denote an arbitrary orientation-preserving diffeomorphism of $\mathbb{T}$ with irrational rotation number. Once and for all we fix a lift $f: \mathbb{R} \rightarrow \mathbb{R}$ of $F$, and to simplify the exposition, we write $\alpha:=\rho(f) \in \mathbb{R} \backslash \mathbb{Q}$.

Using the notation we introduced in $\$ 2.3$ let $\left(a_{n}\right),\left(\alpha_{n}\right),\left(\beta_{n}\right),\left(p_{n}\right),\left(q_{n}\right)$ be the sequences associated to $\alpha$ defined by (2.2), (2.4), (2.5) and (2.8).

For each $n \geq 0$ and $\phi: \mathbb{T} \rightarrow \mathbb{R}$, we define $f_{n}$ and $\phi_{n}$ by

$$
f_{n}:=f^{q_{n}}-p_{n},
$$

and

$$
\phi_{n}:=\mathcal{S}^{q_{n}} \phi=\sum_{i=0}^{q_{n}-1} \phi \circ f^{i} .
$$


And for any $x \in \mathbb{R}$ and $n \geq 0$, we consider the following closed intervals:

$$
\begin{aligned}
I_{n}(x) & :=\left[x, f_{n}(x)\right], \\
J_{n}(x) & :=\left[f_{n+1}(x), f_{n}(x)\right], \\
K_{n}(x) & :=\left[f_{n}^{-2}(x), f_{n}(x)\right] .
\end{aligned}
$$

Let us recall that according to our notation conventions (see \$2), we denote intervals of the real line not taking into account the order of their extreme points.

On the other hand, for any $\hat{x} \in \mathbb{T}$, we will write $\hat{I}_{n}(\hat{x}), \hat{J}_{n}(\hat{x})$, and $\hat{K}_{n}(\hat{x})$ to denote the intervals $\pi\left(I_{n}(x)\right), \pi\left(J_{n}(x)\right), \pi\left(K_{n}(x)\right) \subset \mathbb{T}$, respectively, where $x$ is any point in $\pi^{-1}(\hat{x}) \subset \mathbb{R}$.

The reader can find the proof of the following simple and classical result in the book dMvS93. (Lemma 1.3 of Ch. I):

Lemma 4.1. Given any $n \geq 0$ and any $x \in \mathbb{T}$, the interior of the intervals $\hat{I}_{n}(x)$, $\hat{I}_{n}(F(x)), \ldots, \hat{I}_{n}\left(F^{q_{n+1}-1}(x)\right)$ are two-by-two disjoint. In particular, it holds

$$
\begin{aligned}
J_{n}(x) & =I_{n+1}(x) \cup I_{n}(x), \\
K_{n}(x) & =I_{n}\left(f_{n}^{-2}(x)\right) \cup I_{n}\left(f_{n}^{-1}(x)\right) \cup I_{n}(x) .
\end{aligned}
$$

We will need the following notation:

$$
\begin{aligned}
m_{n}(x) & =\left|f_{n}(x)-x\right|=\operatorname{Leb}\left(I_{n}(x)\right), \\
M_{n} & :=\max _{x \in \mathbb{R}} m_{n}(x) .
\end{aligned}
$$

For the sake of completeness, let us recall the Denjoy-Koksma inequality, (see Her79] for instance):

Proposition 4.2 (Denjoy-Koksma Inequality). If $F$ is $C^{0}$ and $\phi: \mathbb{T} \rightarrow \mathbb{R}$ has bounded variation then, for each $n \geq 1$, it holds

$$
\left\|\phi_{n}-q_{n} \int_{\mathbb{T}} \phi \mathrm{d} \mu\right\|_{C^{0}} \leq \operatorname{Var}(\phi),
$$

where $\mu$ denotes the unique $F$-invariant probability measure and $\operatorname{Var}(\phi)$ the total variation of $\phi$ over $\mathbb{T}$.

On the other hand, for every $x \in \mathbb{R}$ and $1 \leq k \leq q_{n+1}$ it holds

$$
\left|\phi_{k}(y)-\phi_{k}(z)\right| \leq \operatorname{Var}(\phi), \quad \forall y, z \in I_{n}(x) .
$$

\section{1 $C^{r}$-estimates for the log-derivative cocycle}

When $F$ is $C^{1}$, we can consider a very particular cocycle which plays a fundamental role in the analysis of the dynamical properties of $F$ : this is $\log D F=\log D f \in C^{0}(\mathbb{T})$ and will be called the log-derivative cocycle.

The fundamental property of the log-derivative cocycle that turns it to be so important is the chain rule:

$$
\mathcal{S}^{k}(\log D f)=\log D f^{k}, \quad \forall k \geq 1 .
$$

Applying Proposition 4.2 for the log-derivative cocycle we can easily show: 
Corollary 4.3. If $f$ is $C^{2}$, for every $n \geq 2$ we have

$$
\max \left\{\left\|\log D f_{n}\right\|_{C^{0}},\left\|\log D f_{n}^{-1}\right\|_{C^{0}}\right\} \leq \operatorname{Var}(\log D f) .
$$

And on the other hand, for every $x \in \mathbb{R}$ it holds

$$
C^{-1}<\frac{m_{n}(x)}{m_{n}(y)}<C, \quad \forall y \in K_{n-1}(x),
$$

where $C:=\exp (3 \operatorname{Var}(\log D f))$.

The following tree propositions are due to Yoccoz [Yoc84]:

Proposition 4.4. Let $f$ be $C^{2}$. Then, for every $\ell \geq 1, n \geq 2$ and $1 \leq k \leq q_{n}$ it holds

$$
\sum_{i=0}^{k-1}\left(D f^{i}(x)\right)^{\ell} \leq C \frac{M_{n-1}^{\ell-1}}{m_{n-1}(x)^{\ell}}, \quad \forall x \in \mathbb{T},
$$

where $C=C(f):=\exp (\operatorname{Var}(\log D f))$.

Proposition 4.5. Let $f$ be $C^{r}$, with $r \geq 3$, and $s$ be a natural number satisfying $1 \leq s \leq r-1$. Then, there exists a real constant $C>0$, depending only on $f$ and $s$, such that for every $n \geq 2$ and $1 \leq k \leq q_{n}$ it holds

$$
\left|D^{s}\left(\mathcal{S}^{k} \log D f\right)(x)\right|=\left|D^{s} \log D f^{k}(x)\right| \leq C\left(\frac{\sqrt{M_{n-1}}}{m_{n-1}(x)}\right)^{s}, \quad \forall x \in \mathbb{T} .
$$

Proposition 4.6. Supposing $f$ is $C^{3}$, for every $n \geq 2$ and any $x \in \mathbb{R}$ there exist $y \in I_{n-1}(x)$ and $z \in I_{n}(x)$ such that

$$
m_{n}(y)=\frac{\beta_{n}}{\beta_{n-1}} m_{n-1}(z)=\alpha_{n} m_{n-1}(z) .
$$

We will also need the following estimate which, at some extend, can be considered as an improvement of Proposition 4.5 for $k=q_{n}$ :

Proposition 4.7. Let us assume $f$ is $C^{r}$, with $r \geq 3$. Then there exists a constant $C>0$ and a natural number $n_{0}$ such that for any $x \in \mathbb{R}$, every $n \geq n_{0}$ and $0 \leq s \leq r-2$ it holds

$$
\left|D^{s} \log D f_{n}(y)\right| \leq C \frac{\sqrt{M_{n-1}}}{\left(m_{n-1}(x)\right)^{s}}\left[\left(\sqrt{M_{n-1}}\right)^{r-2}+\frac{m_{n}(x)}{m_{n-1}(x)}\right],
$$

for every $y \in K_{n-1}(x)$.

Proof. See $\S 3.6$ of Chapter III in [Yoc95].

The following elementary formula relates the derivatives of the Birkhoff sums of the log-derivative cocycles with the derivatives of the iterates of the diffeomorphism. It can be found in Yoc84, too: 
Proposition 4.8. Given any $r \in \mathbb{N}_{0}$ and $g \in \operatorname{Diff}_{+}^{r+1}(\mathbb{R})$, we have

$$
D^{r+1} g=P_{r}\left(D \log D g, D^{2} \log D g, \ldots, D^{r} \log D g\right) D g,
$$

where $P_{r}$ is the polynomial in $\mathbb{Z}\left[X_{1}, \ldots, X_{r}\right]$ defined inductively by $P_{0}=1$ and

$$
P_{r+1}\left(X_{1}, \ldots, X_{r+1}\right):=X_{1} P_{r}\left(X_{1}, \ldots, X_{r}\right)+\sum_{i=1}^{r} X_{i+1} \frac{\partial P_{r}}{\partial X_{i}}\left(X_{1}, \ldots, X_{r}\right),
$$

for every $r \geq 0$.

In particular, all the polynomials $P_{r}$ satisfy

$$
P_{r}\left(t X_{1}, t^{2} X_{2}, t^{3} X_{3}, \ldots, t^{r} X_{r}\right)=t^{r} P_{r}\left(X_{1}, \ldots, X_{r}\right) .
$$

As a straightforward consequence of propositions 4.5 and 4.8 , we get

Corollary 4.9. Given $f, s, n$ and $k$ as in Proposition 4.5, there exists a constant $C>0$, depending only on $f$ and $s$, such that

$$
\left|D^{s} f^{k}(x)\right| \leq C\left(\frac{\sqrt{M_{n}}}{m_{n}(x)}\right)^{s-1} D f^{k}(x), \quad \forall x \in \mathbb{T} .
$$

\section{2 $C^{r}$-estimates for arbitrary real cocycles}

In this paragraph we shall concern with arbitrary real cocycles and get some estimates for the (higher order) derivatives of them. The main difference with the results of Yoccoz we recalled in 4.2 is that his estimates hold in the whole circle and ours are rather "local".

Now we can get our first $C^{1}$ estimate for real cocycles:

Proposition 4.10. Let $f$ be $C^{2}$. Then, there exists a constant $C>0$ such that for every $\phi \in C^{1}(\mathbb{T})$, any $n \geq 0$, any $x^{\star} \in \mathbb{R}$ satisfying $m_{n}\left(x^{\star}\right)=M_{n}$, and every $y \in K_{n}\left(x^{\star}\right)$, it holds

$$
\left|D\left(\mathcal{S}^{k} \phi\right)(y)\right| \leq C \frac{\|D \phi\|_{C^{0}}}{M_{n}}, \quad \text { for } k=1, \ldots, q_{n+1} .
$$

Proof. Applying estimate (4.6) to the log-derivative cocycle we get

$$
\left|\log D f^{i}(y)-\log D f^{i}(z)\right| \leq \operatorname{Var}(\log D f),
$$

for every $x \in \mathbb{R}$, every $y, z \in I_{n}(x)$ and $0 \leq i \leq q_{n+1}$. This clearly implies,

$$
C^{-1}<\frac{D f^{i}(y)}{D f^{i}(z)}<C, \quad \forall y, z \in K_{n}(x)
$$

where $C:=\exp \left(3\|D \log D f\|_{L^{1}(\mathbb{T})}\right)$. 
Then, combining Proposition 4.4 and estimate (4.8) we get, for every $y \in K_{n}\left(x^{\star}\right)$ and $1 \leq k \leq q_{n+1}$,

$$
\begin{aligned}
\left|D\left(\mathcal{S}^{k} \phi\right)(y)\right| & =\left|\sum_{i=0}^{k-1} D \phi\left(f^{i}(y)\right) D f^{i}(y)\right| \leq\|D \phi\|_{C^{0}} \sum_{i=0}^{k-1} D f^{i}(y) \\
& \leq C\|D \phi\|_{C^{0}} \sum_{i=0}^{k-1} D f^{i}\left(x^{\star}\right) \leq C \frac{\|D \phi\|_{C_{0}}}{m_{n}\left(x^{\star}\right)}=C \frac{\|D \phi\|_{C^{0}}}{M_{n}} .
\end{aligned}
$$

Now let us recall the classical Faa-di Bruno equation:

Proposition 4.11. Given $g, h \in C^{r}(\mathbb{R})$, with $r \geq 1$, it holds

$$
D^{r}(g \circ h)=\sum_{j=1}^{r}\left(D^{j} g \circ h\right) B_{r, j}\left(D^{1} h, \ldots, D^{r-j+1} h\right),
$$

where $B_{r, j}$ is polynomial in $r-j+1$ variables given by

$$
\begin{aligned}
& B_{r, j}\left(x_{1}, \ldots, x_{r-j+1}\right)= \\
& \quad \sum_{\left(c_{i}\right) \in \Omega_{r, j}} \frac{r !}{c_{1} ! \ldots c_{r-j+1} !(1 !)^{c_{1}} \ldots((r-j+1) !)^{c_{r-j+1}}} x_{1}^{c_{1}} x_{2}^{c_{2}} \ldots x_{r-j+1}^{c_{r-j+1}},
\end{aligned}
$$

and where

$$
\Omega_{r, j}:=\left\{\left(c_{1}, \ldots, c_{r-j+1}\right) \in \mathbb{N}_{0}^{r-j+1}: \sum i c_{i}=r, \sum c_{i}=j\right\} .
$$

Using the formulas given in Proposition 4.8 and Proposition 4.11, together with Yoccoz' estimate of Proposition 4.5 we can extend the previous result to higher order derivatives:

Proposition 4.12. Let $f$ be $C^{r+1}$ with $r \geq 2$. Then there exists $C>0$ depending only on $f$ and $r$, such that for every $\phi \in C^{r+1}(\mathbb{T})$, every $n \geq 0,1 \leq k \leq q_{n+1}$, and any $x^{\star} \in \mathbb{R}$ satisfying $m_{n}\left(x^{\star}\right)=M_{n}$, it holds

$$
\left|D^{r}\left(\mathcal{S}^{k} \phi\right)(y)\right| \leq C\|\phi\|_{C^{r}}\left(\frac{1}{\sqrt{M_{n}}}\right)^{r+1}, \quad \forall y \in K_{n}\left(x^{\star}\right) .
$$

Proof. The case $r=1$ was already proved in Proposition 4.10, so let us assume $r \geq 2$. Applying Proposition 4.11 and the estimate given by Corollary 4.9, for any $x \in \mathbb{T}$ we 
obtain

$$
\begin{aligned}
\left|D^{r}\left(\mathcal{S}^{k} \phi\right)(x)\right| & =\left|\sum_{i=0}^{k-1} D^{r}\left(\phi \circ f^{i}\right)(x)\right| \\
& =\left|\sum_{i=0}^{k-1} \sum_{j=1}^{r} D^{j} \phi\left(f^{i}(x)\right) B_{r, j}\left(D f^{i}(x), \ldots, D^{r-j+1} f^{i}(x)\right)\right| \\
& \leq \sum_{j=1}^{r}\left\|D^{j} \phi\right\|_{C^{0}} \sum_{i=0}^{k-1}\left|B_{r, j}\left(D f^{i}(x), \ldots, D^{r-j+1} f^{i}(x)\right)\right| \\
& \leq C\|\phi\|_{C^{r}} \sum_{j=1}^{r}\left(\frac{\sqrt{M_{n}}}{m_{n}(x)}\right)^{r-j} \sum_{i=0}^{k-1}\left(D f^{i}(x)\right)^{j} \\
& \leq C\|\phi\|_{C^{r}} \sum_{j=1}^{r}\left(\frac{\sqrt{M_{n}}}{m_{n}(x)}\right)^{r-j} \frac{M_{n}^{j-1}}{m_{n}(x)^{j}} \\
& =C \frac{\|\phi\|_{C^{r}}}{m_{n}(x)^{r}} \sum_{j=1}^{r}\left(\sqrt{M_{n}}\right)^{r+j-2} \leq C \|_{C^{r}} \frac{\left(\sqrt{M_{n}}\right)^{r-1}}{m_{n}(x)^{r}}
\end{aligned}
$$

Finally, if $y \in K_{n}\left(x^{\star}\right)$, by Corollary 4.3 we have $m_{n}(y) \leq C m_{n}\left(x^{\star}\right)=C M_{n}$, where $C$ is any constant bigger than $\exp (3 \operatorname{Var}(\log D f))$. Thus, putting together this last estimate with (4.10) we obtain (4.9).

\section{Fibered $\mathbb{Z}^{2}$-actions and coboundaries}

The main purpose of this section consists in introducing the fibered $\mathbb{Z}^{2}$-actions on $\mathbb{R}^{2}$ which shall play a central role in our renormalization scheme. We will see that (a lift of) a circle diffeomorphism and a cocycle naturally induce a fibered $\mathbb{Z}^{2}$-action. Then, we shall extend the notion of coboundary to fibered $\mathbb{Z}^{2}$-actions and in Lemma 5.1 we prove this new definition indeed generalizes the previous one given in $\$ 2.2$.

Then, in Proposition 5.3 we give a simple but fundamental characterization of certain coboundaries which is mainly inspired in the definition of quasi-rotations of Yoccoz Yoc95.

The space $\mathcal{W}^{r}:=\operatorname{Diff}_{+}^{r}(\mathbb{R}) \times C^{r}(\mathbb{R})$ can be seen as a subgroup of Diff ${ }_{+}^{r}\left(\mathbb{R}^{2}\right)$ defining

$$
(f, \psi):(x, y) \mapsto(f(x), y+\psi(x)),
$$

for each $(f, \psi) \in \mathcal{W}^{r}$.

The space of fibered $\mathbb{Z}^{2}$-actions on $\mathbb{R}^{2}$ will be denoted by

$$
\mathscr{A}^{r}:=\operatorname{Hom}\left(\mathbb{Z}^{2}, \mathcal{W}^{r}\right) \subset \operatorname{Hom}\left(\mathbb{Z}^{2}, \operatorname{Diff}_{+}^{r}\left(\mathbb{R}^{2}\right)\right) .
$$

Given any $\Phi \in \mathscr{A}^{r}$ and any $(m, n) \in \mathbb{Z}^{2}$, we write

$$
\Phi(m, n)=\left(f_{\Phi}^{m, n}, \psi_{\Phi}^{m, n}\right) \in \mathcal{W}^{r} .
$$


Whenever the action is clear from the context, we shall just write $\left(f^{m, n}, \psi^{m, n}\right)$ instead of $\left(f_{\Phi}^{m, n}, \psi_{\Phi}^{m, n}\right)$.

There are two group actions on $\mathscr{A}^{r}$ that will be used in our renormalization scheme: the first one is the left $\mathcal{W}^{s}$-action $\mathcal{T}: \mathcal{W}^{s} \times \mathscr{A}^{r} \rightarrow \mathscr{A}^{r}$ (with $0 \leq s \leq r$ ), given by conjugation in $\operatorname{Diff}_{+}^{r}\left(\mathbb{R}^{2}\right)$, i.e.

$$
\begin{aligned}
\mathcal{T}_{(g, \xi)}(\Phi)(m, n) & :=(g, \xi) \Phi(m, n)(g, \xi)^{-1} \\
& =\left(g f_{\Phi}^{m, n} g^{-1},\left(\psi_{\Phi}^{m, n}+\xi f_{\Phi}^{m, n}-\xi\right) \circ g^{-1}\right)
\end{aligned}
$$

for every $(g, \xi) \in \mathcal{W}^{s}, \Phi \in \mathscr{A}^{r}$ and $(m, n) \in \mathbb{Z}^{2}$. The second one is the left $\mathrm{GL}(2, \mathbb{Z})$ action $\mathcal{U}: \operatorname{GL}(2, \mathbb{Z}) \times \mathscr{A}^{r} \rightarrow \mathscr{A}^{r}$ given by change of basis in $\mathbb{Z}^{2}$, i.e.

$$
\mathcal{U}_{A}(\Phi)(m, n):=\Phi\left(m^{\prime}, n^{\prime}\right)
$$

where $A \in \mathrm{GL}(2, \mathbb{Z})$ and

$$
\left(\begin{array}{c}
m^{\prime} \\
n^{\prime}
\end{array}\right):=A^{-1}\left(\begin{array}{c}
m \\
n
\end{array}\right) .
$$

A very simple but fundamental remark about these actions is that $\mathcal{T}$ and $\mathcal{U}$ commute. Most of the time we will work on the subset $\mathscr{A}_{0}^{r} \subset \mathscr{A}^{r}$ given by

$$
\mathscr{A}_{0}^{r}:=\left\{\Phi \in \mathscr{A}^{r}: \operatorname{Fix}\left(f_{\Phi}^{m, n}\right)=\emptyset, \forall(m, n) \in \mathbb{Z}^{2} \backslash\{(0,0)\}\right\} .
$$

Observe that this subset is invariant under the actions $\mathcal{T}$ and $\mathcal{U}$.

Next, notice that each pair $(f, \phi) \in \overline{\operatorname{Diff}}_{+}^{r}(\mathbb{T}) \times C^{r}(\mathbb{T}) \subset \mathcal{W}^{r}$ naturally induces an action $\Gamma=\Gamma(f, \phi) \in \mathscr{A}^{r}$ given by

$$
\Gamma:\left\{\begin{aligned}
(1,0) & \mapsto(\tau, 0), \\
(0,1) & \mapsto(f, \phi),
\end{aligned}\right.
$$

where $\tau$ is the translation $x \mapsto x-1$. Notice that this action $\Gamma$ belongs to $\mathscr{A}_{0}^{r}$ if and only if $\operatorname{Per}(\pi(f))=\emptyset$, i.e. $\rho(f)$ is an irrational number.

Now, taking into account that a circle diffeomorphism and a cocycle induce a fibered $\mathbb{Z}^{2}$-action, it is reasonable to extend the notion of coboundary to fibered $\mathbb{Z}^{2}$-actions: we say that $\Phi \in \mathscr{A}_{0}^{r}$ is a $C^{s}$-coboundary, with $0 \leq s \leq r$, if and only if there exist $(g, \xi) \in \mathcal{W}^{s}$ and $A \in \mathrm{GL}(2, \mathbb{Z})$ such that $\Phi^{\prime}:=\overline{\mathcal{U}}_{A}\left(\overline{\mathcal{T}}_{(g, \xi)} \Phi\right)$ satisfies the following conditions:

$$
\begin{gathered}
f_{\Phi^{\prime}}^{1,0}=\tau, \\
\psi_{\Phi^{\prime}}^{1,0}=\psi_{\Phi^{\prime}}^{0,1} \equiv 0 .
\end{gathered}
$$

It is very easy to verify that this new notion of coboundary is coherent with the previous one. In fact, we have the following

Lemma 5.1. Let $F \in \operatorname{Diff}_{+}^{r}(\mathbb{T})$ (with $\left.0 \leq r \leq \infty\right)$ be such that $\rho(F) \in(\mathbb{R} \backslash \mathbb{Q}) / \mathbb{Z}$, $\phi \in C^{r}(\mathbb{T})$ and $f \in \widetilde{\operatorname{Diff}_{+}^{r}}(\mathbb{T})$ be any lift of $F$. Then $\phi \in B\left(F, C^{s}(\mathbb{T})\right)$ if and only if the induced action $\Gamma(f, \phi)$ given by (5.1) is a $C^{s}$-coboundary. 
Proof. Let us start assuming that $\Gamma=\Gamma(f, \phi)$ is a $C^{s}$-coboundary. This means there exist $(g, u) \in \mathcal{W}^{s}$ and $A \in \mathrm{GL}(2, \mathbb{Z})$ such that $\Xi:=\mathcal{T}_{(g, u)}\left(\mathcal{U}_{A} \Gamma\right)$ satisfies $\psi_{\Xi}^{1,0}=\psi_{\Xi}^{0,1} \equiv 0$ and $f_{\Xi}^{1,0}=\tau$. This implies that

$$
\psi_{\Xi}^{m, n} \equiv 0, \quad \forall(m, n) \in \mathbb{Z}^{2} .
$$

In particular, if we write $A=\left(\begin{array}{ll}a & c \\ b & d\end{array}\right)$, we get

$$
\begin{gathered}
\psi_{\Xi}^{a, b}=(u \circ \tau-u) \circ g^{-1} \equiv 0 \\
\psi_{\Xi}^{c, d}=(\phi+u \circ f-u) \circ g^{-1} \equiv 0 .
\end{gathered}
$$

By (5.2), $u$ is $\mathbb{Z}$-periodic, and by (5.3) $\phi$ is a $C^{s}$-coboundary for $F$.

Reciprocally, let us suppose $\phi \in B\left(F, C^{s}(\mathbb{T})\right)$. So, we can find $u \in C^{s}(\mathbb{T})$ satisfying $u f-u=\phi$. Thus, writing $\Gamma^{\prime}:=\mathcal{T}_{(i d,-u)} \Gamma$ we clearly have $f_{\Gamma^{\prime}}^{1,0}=f_{\Gamma}^{1,0}=\tau$ and $\psi_{\Gamma^{\prime}}^{1,0}=\psi_{\Gamma^{\prime}}^{0,1} \equiv 0$. Therefore, $\Gamma$ is a $C^{s}$-coboundary.

Our next result is a very elementary but useful characterization of coboundaries that will turn to be our fundamental tool to construct a coboundary as a small perturbation of a cocycle after applying our renormalization scheme. However, first we need a very simple (and well-known) lemma about cohomological equations on the real line:

Lemma 5.2. Given any $f \in \operatorname{Diff}_{+}^{r}(\mathbb{R})$ with $\operatorname{Fix}(f)=\emptyset$ and any $\phi \in C^{r}(\mathbb{R})$, the cohomological equation

$$
u f-u=\phi
$$

always admits a solution $u \in C^{r}(\mathbb{R})$.

Proof. Let us write $z:=f(0)$. Since $f$ is fixed-point free, we do not loose any generality assuming $z>0$.

Next, let $u:[0, z] \rightarrow \mathbb{R}$ be any $C^{r}$ function satisfying

$$
\left.u\right|_{\left[0, \frac{z}{3}\right]} \equiv 0,\left.\quad u\right|_{\left[\frac{2 z}{3}, z\right]} \equiv \phi
$$

Now, since the interval $[0, z]$ is a fundamental domain for $f$, for any $x \in \mathbb{R}$ there is a unique $n(x) \in \mathbb{Z}$ such that $f^{-n(x)}(x) \in\left[0, x_{1}\right)$, and so we can extend the function $u$ to the whole real line by writing

$$
u(x):=u\left(f^{-n(x)}(x)\right)+\mathcal{S}^{n(x)} \phi\left(f^{-n(x)}(x)\right), \quad \forall x \in \mathbb{R} .
$$

By the very definition, $u$ is a $C^{r}$ function and satisfies $u f-u=\phi$.

Now we can state our characterization of coboundaries within the context of fibered $\mathbb{Z}^{2}$-actions:

Proposition 5.3. Let $\Phi \in \mathscr{A}_{0}^{r}$ be so that there exists $x^{\star} \in \mathbb{R}$ satisfying

$$
\begin{aligned}
& \psi^{1,0}(x)=0, \quad \forall x \in\left[x^{\star}, f^{0,1}\left(x^{\star}\right)\right], \\
& \psi^{0,1}(x)=0, \quad \forall x \in\left[x^{\star}, f^{1,0}\left(x^{\star}\right)\right] .
\end{aligned}
$$

Then, $\Phi$ is a $C^{r}$-coboundary. 
Proof. First of all, notice that we do not loose any generality supposing

$$
f^{0,1}\left(x^{\star}\right) \in\left(f^{-1,0}\left(x^{\star}\right), f^{1,0}\left(x^{\star}\right)\right) .
$$

In this case there are two possibilities: $f^{0,1}\left(x^{\star}\right)$ belongs either to $\left(x^{\star}, f^{1,0}\left(x^{\star}\right)\right)$, or to $\left(f^{-1,0}\left(x^{\star}\right), x^{\star}\right)$. Let us suppose the first case holds, being the second one completely analogous.

By Lemma 5.2, we can find a function $u \in C^{r}(\mathbb{R})$ such that

$$
\psi^{1,0}=u f^{1,0}-u \text {. }
$$

Notice that by (5.4), we have

$$
u\left(f^{1,0}(x)\right)=u(x), \quad \forall x \in\left[x^{\star}, f^{0,1}\left(x^{\star}\right)\right] .
$$

Now, since we are supposing that $\left[x^{\star}, f^{0,1}\left(x^{\star}\right)\right] \subset\left[x^{\star}, f^{1,0}\left(x^{\star}\right)\right]$, from (5.7) we can conclude there exists a unique function $\bar{u} \in C^{r}(\mathbb{R})$ satisfying

$$
\begin{aligned}
\left.\bar{u}\right|_{\left[x^{\star}, f^{1,0}\left(x^{\star}\right)\right]} & \left.\equiv u\right|_{\left[x^{\star}, f^{1,0}\left(x^{\star}\right)\right]}, \\
\bar{u}\left(f^{1,0}(x)\right) & =\bar{u}(x), \quad \forall x \in \mathbb{R} .
\end{aligned}
$$

Next, if we define

$$
\bar{\psi}:=\psi^{0,1}+u-u f^{0,1} \in C^{r}(\mathbb{R}),
$$

it can be easily shown that $\bar{\psi}$ is $f^{1,0}$-periodic. In fact, since $\left(f^{1,0}, \psi^{1,0}\right)$ and $\left(f^{0,1}, \psi^{0,1}\right)$ commute, we have

$$
\psi^{1,1}=\psi^{1,0}+\psi^{0,1} \circ f^{1,0}=\psi^{0,1}+\psi^{1,0} \circ f^{0,1},
$$

and so,

$$
\begin{aligned}
\bar{\psi} f^{1,0} & =\left(\psi^{0,1}+u-u f^{0,1}\right) f^{1,0}=\psi^{0,1} f^{1,0}+u f^{1,0}-u+u-u f^{1,1} \\
& =\psi^{0,1} f^{1,0}+\psi^{1,0}+u-u f^{1,1}=\psi^{0,1}+\psi^{1,0} f^{0,1}+u-u f^{1,1} \\
& =\psi^{0,1}+\left(u f^{1,0}-u\right) f^{0,1}+u-u f^{1,1}=\psi^{0,1}+u-u f^{0,1} \\
& =\bar{\psi}
\end{aligned}
$$

Now, combining (5.5), (5.8) and (5.10) we can conclude that

$$
\bar{\psi}=\bar{u}-\bar{u} f^{0,1} \text {. }
$$

Then, taking into account equations (5.6), (5.9) and (5.11) we can easily see that

$$
\mathcal{T}_{(i d, \bar{u}-u)} \Phi(\mathbf{i})=\left(f^{\mathbf{i}}, 0\right), \quad \text { for } \mathbf{i}=(1,0),(0,1) .
$$

Finally, applying Lemma 5.2 one again we can construct an orientation-preserving $C^{r}$ diffeomorphism $h: \mathbb{R} \rightarrow \mathbb{R}$ satisfying $h f^{1,0} h^{-1}=\tau$. Thus,

$$
\mathcal{T}_{(h, \bar{u}-u)} \Phi=\left\{\begin{array}{l}
(1,0) \mapsto(\tau, 0), \\
(0,1) \mapsto\left(h \circ f^{0,1} \circ h^{-1}, 0\right),
\end{array}\right.
$$

and the proposition is proved. 


\section{Renormalization of fibered $\mathbb{Z}^{2}$-actions}

The main aim of this section is to introduce the renormalization scheme for $\mathbb{Z}^{2}$-actions and to show how it can be used to construct coboundaries by perturbation of the original cocycle. Indeed, the notion of coboundary (for $\mathbb{Z}^{2}$-actions) turns out to be expressly renormalization-invariant, which allows us to take advantage of the smoothing effect of renormalization.

As in $\oiint, F$ will denote an arbitrary minimal $C^{r}$ diffeomorphism of $\mathbb{T}$ (with $r \geq 3$ ), $f \in \widetilde{\operatorname{Diff}_{+}^{r}}(\mathbb{T})$ a lift of $F$, and $\phi: \mathbb{T} \rightarrow \mathbb{R}$ an arbitrary $C^{r}$ real cocycle.

To simplify the notation, we write $\alpha=\rho(f)$, and since we are assuming $\alpha \in \mathbb{R} \backslash \mathbb{Q}$, we consider the sequences $\left(a_{n}\right),\left(\alpha_{n}\right),\left(\beta_{n}\right),\left(p_{n}\right),\left(q_{n}\right)$ associated to $\alpha$ defined by (2.2), (2.4), (2.5), and (2.8).

Then, we define the matrices

$$
A_{n}=A_{n}(\alpha):=(-1)^{n}\left(\begin{array}{cc}
q_{n} & -p_{n} \\
-q_{n-1} & p_{n-1}
\end{array}\right), \quad \forall n \geq-1 .
$$

Notice that, by (2.7), all the matrices $A_{n}$ belong to $\operatorname{GL}(2, \mathbb{Z})$.

Now, for each $n \geq-1$ we define the $n^{\text {th }}$-renormalized action $\Gamma_{n}(\phi)$ by

$$
\Gamma_{n}(\phi):=\mathcal{U}_{A_{n}}(\Gamma(f, \phi)), \quad \forall n \geq-1,
$$

where, of course, $\Gamma(f, \phi)$ denotes the induced action defined by (5.1). Notice that

$$
\Gamma_{n}(\phi):\left\{\begin{array}{l}
(1,0) \mapsto\left(f_{n-1}, \phi_{n-1}\right)=\left(f^{q_{n-1}}-p_{n-1}, \mathcal{S}_{f}^{q_{n-1}} \phi\right), \\
(0,1) \mapsto\left(f_{n}, \phi_{n}\right)=\left(f^{q_{n}}-p_{n}, \mathcal{S}_{f}^{q_{n}} \phi\right) .
\end{array}\right.
$$

Lemma 6.1. Let $n \geq 3$ and $x^{\star} \in \mathbb{R}$ be arbitrary. Then, there exists $u \in C^{r}(\mathbb{T})$ such that the cocycle $\bar{\phi}:=\phi+u-u F$ satisfies

$$
\bar{\phi}_{n-1}(y)=0, \quad \forall y \in J_{n-1}\left(x^{\star}\right) .
$$

Moreover, there exists a real constant $C>0$ depending only on $F$ and $r$, such that whenever $x^{\star}$ satisfies $m_{n-1}\left(x^{\star}\right)=M_{n-1}$, the function $u$ can be chosen fulfilling the following estimate:

$$
\left|D^{r} u(y)\right| \leq C\left\|\left.\phi_{n-1}\right|_{K_{n-1}\left(x^{\star}\right)}\right\|_{C^{r}} \Theta(\alpha, n, r)\left(\frac{1}{M_{n-1}}\right)^{r}
$$

for every $y \in J_{n-1}\left(x^{\star}\right)$, where

$$
\Theta(\alpha, n, r):=\sum_{i=0}^{r}\left(\frac{\beta_{n-1}}{\beta_{n-1}-\beta_{n}}\right)^{i} .
$$

Proof. First, let $\zeta: \mathbb{R} \rightarrow \mathbb{R}$ be any auxiliary smooth function satisfying:

- $\zeta(x)=0$, for every $x \leq 0$; 
- $0<\zeta(x)<1$, for every $x \in(0,1)$;

- $\zeta(x)=1$, for every $x \geq 1$.

Let $\hat{x}:=\pi\left(x^{\star}\right) \in \mathbb{T}$. By Lemma 4.1 we know that, $\hat{I}_{n}(\hat{x}) \cap \hat{I}_{n-1}(\hat{x})=\{\hat{x}\}$ and $F^{q_{n}+q_{n-1}}(\hat{x})$ belongs to the interior of $\hat{I}_{n-1}(\hat{x})$. In particular, this implies that $I_{n}\left(x^{\star}\right)$ and $f_{n-1}\left(I_{n}\left(x^{\star}\right)\right)$ are disjoint, and the last interval is contained in $I_{n-1}\left(x^{\star}\right)$.

We can assume $n$ is odd (the other case is completely analogous), so it holds

$$
f_{n}\left(x^{\star}\right)<x^{\star}<f_{n-1}\left(f_{n}\left(x^{\star}\right)\right)=f_{n}\left(f_{n-1}\left(x^{\star}\right)\right)<f_{n-1}\left(x^{\star}\right) .
$$

Notice since $\Gamma_{n}(\phi) \in \mathscr{A}_{0}^{r}$, the previous relation holds for every $x \in \mathbb{R}$.

Now, we define $u: J_{n-1}\left(x^{\star}\right) \rightarrow \mathbb{R}$ by

$$
u(y):=\zeta\left(\frac{y-x^{\star}}{f_{n}\left(f_{n-1}\left(x^{\star}\right)\right)-x^{\star}}\right) \phi_{n-1}\left(f_{n-1}^{-1}(y)\right),
$$

for every $y \in J_{n-1}\left(x^{\star}\right)=\left[f_{n}\left(x^{\star}\right), f_{n-1}\left(x^{\star}\right)\right]$. Then, it clearly holds

$$
u\left(f_{n-1}(y)\right)-u(y)=\phi_{n-1}(y),
$$

whenever $y$ and $f_{n-1}(y)$ both belong to $J_{n-1}\left(x^{\star}\right)$, i.e. for every $y \in I_{n}\left(x^{\star}\right)$.

Now, we can extend our function $u$ to the whole real line $\mathbb{R}$ to get a $C^{r} \mathbb{Z}$-periodic function satisfying

$$
u\left(F^{q_{n-1}}(y)\right)-u(y)=\phi_{n-1}(y), \quad \forall y \in \hat{J}_{n-1}(\hat{x}) .
$$

Notice that if we write $\bar{\phi}:=\phi+u-u F$, we clearly get

$$
\bar{\phi}_{n-1}(y)=\mathcal{S}_{f}^{q_{n-1}} \bar{\phi}(y)=\phi_{n-1}(y)+u(y)-u\left(f_{n-1}(y)\right)=0,
$$

for every $y \in J_{n-1}\left(x^{\star}\right)$, as desired.

So, it remains to prove $u$ satisfies estimate (6.4). To do this, first notice that combining Corollary 4.3 and Proposition 4.6 we get

$$
C^{-1} \frac{\beta_{n}}{\beta_{n-1}} \leq \frac{m_{n}\left(f_{n-1}\left(x^{\star}\right)\right)}{m_{n-1}\left(x^{\star}\right)} \leq C \frac{\beta_{n}}{\beta_{n-1}}
$$

where $C>1$ is a constant which depends on $F$, but does not either on $n$ or $x^{\star}$.

Now, to simplify the notation let us write

$$
\ell:=\left|f_{n}\left(f_{n-1}\left(x^{\star}\right)\right)-x^{\star}\right| .
$$

Observe that, since we are assuming $n$ is odd, we have $\ell=m_{n-1}\left(x^{\star}\right)-m_{n}\left(f_{n-1}\left(x^{\star}\right)\right)$. Hence, by (6.8) it holds

$$
\ell \geq\left(1-C^{-1} \frac{\beta_{n}}{\beta_{n-1}}\right) m_{n-1}\left(x^{\star}\right) \geq C^{-1} \frac{\beta_{n-1}-\beta_{n}}{\beta_{n-1}} M_{n-1} .
$$


Now, invoking Denjoy-Koksma inequality (Proposition 4.2), Corollary 4.9. Faa-di Bruno formula (Proposition 4.11), Proposition 4.12 and estimate (6.9), we can prove (6.4). In fact, for any $y \in J_{n-1}\left(x^{\star}\right) \subset K_{n-1}\left(x^{\star}\right)$ we have

$$
\begin{aligned}
& \left|D^{r} u(y)\right|=\left|\sum_{i=0}^{r}\left(\begin{array}{c}
r \\
i
\end{array}\right) D^{i} \zeta\left(\frac{y-x^{\star}}{\ell}\right) \ell^{-i} D^{r-i}\left(\phi_{n-1} \circ f_{n-1}^{-1}\right)(y)\right| \\
& \leq C \sum_{i=0}^{r}\left(\frac{\beta_{n-1}}{\left(\beta_{n-1}-\beta_{n}\right) M_{n-1}}\right)^{i} \mid \sum_{j=1}^{r-i}\left(D^{j} \phi_{n-1}\right)\left(f_{n-1}^{-1}(y)\right) \\
& B_{r-i, j}\left(D^{1} f_{n-1}^{-1}, \ldots, D^{r-i-j+1} f_{n-1}^{-1}\right) \mid \\
& \leq C\left\|\left.\phi_{n-1}\right|_{K_{n-1}\left(x^{\star}\right)}\right\|_{C^{r}} \Theta(\alpha, n, r) \sum_{i=0}^{r}\left(\frac{1}{M_{n-1}}\right)^{i} \\
& \leq C\left\|\left.\phi_{n-1}\right|_{K_{n-1}\left(x^{\star}\right)}\right\|_{C^{r}} \Theta(\alpha, n, r)\left(\frac{1}{M_{n-1}}\right)^{r-i}\left(D f_{n-1}^{-1}(y)\right)^{j}\left(\frac{\sqrt{M_{n-1}}}{m_{n-1}(y)}\right)^{r-i-j}
\end{aligned}
$$

and estimate (6.4) is proved.

Lemma 6.2. Let $\phi, x^{\star}, n, u$ and $\bar{\phi}$ be as in Lemma 6.1. Then there exists $\xi \in C^{r}(\mathbb{T})$ such that

$$
\begin{aligned}
& \operatorname{supp} \xi \subset \hat{I}_{n-1}\left(\pi\left(x^{\star}\right)\right) \cup \hat{I}_{n-1}\left(\pi\left(f_{n-1}\left(x^{\star}\right)\right)\right), \\
& \xi(y)+\xi\left(f_{n-1}(y)\right)=\bar{\phi}_{n}(y), \quad \forall y \in I_{n-1}\left(x^{\star}\right) .
\end{aligned}
$$

Moreover, there exists a constant $C>0$ depending only on $F$ and $r$ such that the function $\xi$ can constructed fulfilling the following estimate:

$$
\|\xi\|_{C^{r}} \leq C\left\|\left.\bar{\phi}_{n}\right|_{I_{n-1}\left(x^{\star}\right)}\right\|_{C^{r}}\left(\frac{1}{M_{n-1}}\right)^{r} .
$$

Proof. As in the proof of Lemma 6.1, we will assume $n$ is odd, and therefore, for every $x \in \mathbb{R}$ it holds $f_{n}(x)<x<f_{n-1}(x)<f_{n-1}^{2}(x)$.

Then, let us start defining $\xi$ on the interval $\left[x^{\star}, f_{n-1}^{2}\left(x^{\star}\right)\right]=I_{n-1}\left(x^{\star}\right) \cup I_{n-1}\left(f_{n-1}\left(x^{\star}\right)\right)$ by writing

$$
\xi(y):= \begin{cases}\zeta\left(\frac{y-x^{\star}}{f_{n-1}\left(x^{\star}\right)-x^{\star}}\right) \bar{\phi}_{n}(y), & \text { if } y \in I_{n-1}\left(x^{\star}\right), \\ {\left[1-\zeta\left(\frac{f_{n-1}^{-1}(y)-x^{\star}}{f_{n-1}\left(x^{\star}\right)-x^{\star}}\right)\right] \bar{\phi}_{n}\left(f_{n-1}^{-1}(y)\right),} & \text { if } y \in I_{n-1}\left(f_{n-1}\left(x^{\star}\right)\right),\end{cases}
$$

where $\zeta$ is the auxiliary function we used in the proof of Lemma 6.1 
In this way, our function $\xi$ is clearly $C^{r}$ on the interiors of the intervals $I_{n-1}\left(x^{\star}\right)$ and $I_{n-1}\left(f_{n-1}\left(x^{\star}\right)\right)$, and by the very properties of the auxiliary function $\zeta$, we have

$$
D^{k} \xi\left(x^{\star}\right)=D^{k} \xi\left(f_{n-1}^{2}\left(x^{\star}\right)\right)=0, \quad \text { for } k=0,1, \ldots, r .
$$

In order to see that $\xi$ is also continuous and has continuous derivatives up to order $r$ at $f_{n-1}\left(x^{\star}\right)$, let us consider the fibered $\mathbb{Z}^{2}$-action $\Phi:=\Gamma_{n}(\bar{\phi})$ (see (6.2) for the definition of $\Gamma_{n}$ ) and notice that condition (6.3) can be translated into the $\mathbb{Z}^{2}$-action language stating

$$
\psi_{\Phi}^{1,0}(y)=0, \quad \forall y \in J_{n-1}\left(x^{\star}\right)=\left[f_{n}\left(x^{\star}\right), f_{n-1}\left(x^{\star}\right)\right] .
$$

On the other hand, since $\Phi(1,0)$ and $\Phi(0,1)$ commute in $\operatorname{Diff}_{+}^{r}\left(\mathbb{R}^{2}\right)$, and taking into account that $f_{\Phi}^{1,0}=f_{n-1}$ and $f_{\Phi}^{0,1}=f_{n}$, we have

$$
\psi_{\Phi}^{0,1}(y)+\psi_{\Phi}^{1,0}\left(f_{n}(y)\right)=\psi_{\Phi}^{1,0}(y)+\psi_{\Phi}^{0,1}\left(f_{n-1}(y)\right), \quad \forall y \in \mathbb{R}
$$

Now, putting together equations (6.16) and (6.17), and recalling that $\psi_{\Phi}^{0,1}=\bar{\phi}_{n}$, we conclude that

$$
\bar{\phi}_{n}(y)=\bar{\phi}_{n}\left(f_{n-1}(y)\right), \quad \forall y \in I_{n-1}\left(x^{\star}\right),
$$

From (6.18) we can easily show that $\xi$ is continuous and has continuous derivatives up to order $r$ at the point $f_{n-1}\left(x^{\star}\right)$.

Hence, by this remark and (6.15) we can affirm there is a unique extension of $\xi$ to the whole real line such that it is $C^{r}, \mathbb{Z}$-periodic and satisfies

$$
\operatorname{supp} \xi \subset \bigcup_{k \in \mathbb{Z}}\left[x^{\star}+k, f_{n-1}^{2}\left(x^{\star}\right)+k\right]
$$

Of course, this is clearly equivalent to (6.11). The condition (6.12) is also satisfied by the pure construction of $\xi$.

Next, let us prove that $\xi$ satisfies estimate (6.13). To do this, first let $y$ be an arbitrary point in $I_{n-1}\left(x^{\star}\right)$ and notice that

$$
\begin{aligned}
\left|D^{r} \xi(y)\right| & =\left|\sum_{j=0}^{r}\left(\begin{array}{l}
r \\
j
\end{array}\right)\left(D^{j} \zeta\right)\left(\frac{y-x^{\star}}{f_{n-1}\left(x^{\star}\right)-x^{\star}}\right)\left(\frac{1}{M_{n-1}}\right)^{j} D^{r-j} \bar{\phi}_{n}(y)\right| \\
& \leq C\left(\frac{1}{M_{n-1}}\right)^{r}\left\|\left.\bar{\phi}_{n}\right|_{I_{n-1}\left(x^{\star}\right)}\right\|_{C^{r}}
\end{aligned}
$$

Now, if $y$ denotes an arbitrary point of $I_{n-1}\left(f_{n-1}\left(x^{\star}\right)\right)$ and $1 \leq i \leq r$, by Corol- 
lary 4.9 and Proposition 4.11 we have

$$
\begin{aligned}
& \left|D^{i}\left[\zeta\left(\frac{f_{n-1}^{-1}(y)-x^{\star}}{f_{n-1}\left(x^{\star}\right)-x^{\star}}\right)\right]\right| \\
& \quad=\mid \sum_{j=1}^{i}\left(D^{j} \zeta\right)\left(\frac{f_{n-1}^{-1}(y)-x^{\star}}{f_{n-1}\left(x^{\star}\right)-x^{\star}}\right)\left(\frac{1}{M_{n-1}}\right)^{j} \\
& \quad B_{i, j}\left(D f_{n-1}^{-1}(y), \ldots, D^{i-j+1} f_{n-1}^{-1}(y)\right) \mid \\
& \quad \leq C \sum_{j=1}^{i}\left(\frac{1}{M_{n-1}}\right)^{j}\left(D f_{n-1}^{-1}(y)\right)^{j}\left(\frac{\sqrt{M_{n-1}}}{m_{n-1}(y)}\right)^{i-j} \\
& \quad \leq C \sum_{j=1}^{i}\left(\frac{1}{\sqrt{M_{n-1}}}\right)^{i+j} \leq C\left(\frac{1}{M_{n-1}}\right)^{i},
\end{aligned}
$$

and so,

$$
\begin{aligned}
\left|D^{r} \xi(y)\right| & \leq C \sum_{i=0}^{r}\left|D^{i}\left[\zeta\left(\frac{f_{n-1}^{-1}(y)-x^{\star}}{f_{n-1}\left(x^{\star}\right)-x^{\star}}\right)\right] D^{r-i} \bar{\phi}_{n}\left(f_{n-1}^{-1}(y)\right)\right| \\
& \leq C \sum_{i=0}^{r}\left(\frac{1}{M_{n-1}}\right)^{i}\left\|\left.\bar{\phi}_{n} \circ f_{n-1}^{-1}\right|_{I_{n-1}\left(f_{n-1}\left(x^{\star}\right)\right)}\right\|_{C^{r-i}} \\
& \leq C\left(\frac{1}{M_{n-1}}\right)^{r}\left\|\left.\bar{\phi}_{n} \circ f_{n-1}^{-1}\right|_{I_{n-1}\left(f_{n-1}\left(x^{\star}\right)\right)}\right\|_{C^{r}} \\
& =C\left(\frac{1}{M_{n-1}}\right)^{r}\left\|\left.\bar{\phi}_{n}\right|_{I_{n-1}\left(x^{\star}\right)}\right\|_{C^{r}} .
\end{aligned}
$$

where the last equality is consequence of (6.18).

Now, combining (6.19) and (6.21) we can easily get (6.13).

Lemma 6.3. Let $\phi, \bar{\phi}$ and $\xi$ be as in Lemma 6.2. Then, the cocycle $\tilde{\phi}:=\phi-\xi$ is a $C^{r}$-coboundary for $F$.

Proof. Since $\phi$ and $\bar{\phi}$ are $C^{r}$-cohomologous, this is equivalent to show that $\bar{\phi}-\xi \in$ $B\left(F, C^{r}(\mathbb{T})\right)$. To do this, we will show that the $\mathbb{Z}^{2}$-action $\Gamma:=\Gamma_{n}(\bar{\phi}-\xi)$ is a $C^{r}$ coboundary.

First observe that $\Gamma(1,0)=\left(f_{n-1}, \bar{\phi}_{n-1}-\xi_{n-1}\right)$. By (6.3) we know $\left.\bar{\phi}_{n-1}\right|_{I_{n}\left(x^{\star}\right)} \equiv 0$, and if we write $\hat{x}:=\pi\left(x^{\star}\right)$, Lemma 4.1 implies that for any $y \in \hat{I}_{n}(\hat{x})$, it holds

$$
F^{i}(y) \notin \hat{I}_{n-1}(\hat{x}) \backslash\{\hat{x}\}, \quad \text { for } i=0,1, \ldots, q_{n-1}-1 .
$$

On the other hand, we affirm that

$$
F^{i}(y) \notin \hat{I}_{n-1}\left(F^{q_{n-1}}(\hat{x})\right), \quad \text { for } i=0,1, \ldots, q_{n-1}-1 .
$$


In fact, let us suppose that (6.23) does not hold. So, there exists $y \in \hat{I}_{n}(\hat{x})$ and $i \in\left\{1, \ldots, q_{n-1}-1\right\}$ such that $F^{i}(y) \in \hat{I}_{n-1}\left(F^{q_{n-1}}(\hat{x})\right)$.

Moreover, we have

$$
F^{q_{n-1}-i}\left(F^{i}(y)\right)=F^{q_{n-1}}(y) \in F^{q_{n-1}}\left(\hat{I}_{n}(\hat{x})\right)=\hat{I}_{n}\left(F^{q_{n-1}}(\hat{x})\right),
$$

and by Lemma 4.1 we know that $F^{q_{n-1}-i}\left(F^{2 q_{n-1}}(\hat{x})\right) \notin \hat{I}_{n-1}\left(F^{q_{n-1}}(\hat{x})\right)$. In particular, this last remark and (6.24) imply that $F^{q_{n-1}-i}\left(F^{2 q_{n-1}}(\hat{x})\right) \in I_{n}\left(F^{q_{n-1}}(\hat{x})\right)$, and since $F$ is topologically conjugate to the irrational rotation $R_{\alpha}$, this clearly contradicts (2.6). Hence, (6.23) is proved.

Now, by (6.11), (6.22) and (6.23), we have $\left.\xi_{n-1}\right|_{I_{n}\left(x^{\star}\right)} \equiv 0$, and so,

$$
\psi_{\Gamma}^{1,0}(y)=\bar{\phi}_{n-1}(y)=0, \quad \forall y \in I_{n}\left(x^{\star}\right)=\left[x^{\star}, f_{\Gamma}^{0,1}\left(x^{\star}\right)\right] .
$$

On the other hand, let $z$ be an arbitrary point in $\hat{I}_{n-1}(\hat{x})$ and let us consider the set

$$
A_{z}:=\left\{i \in \mathbb{N}_{0}: F^{i}(z) \in \hat{I}_{n-1}(\hat{x}) \cup \hat{I}_{n-1}\left(F^{q_{n-1}}(\hat{x})\right), i<q_{n}\right\} .
$$

Let us prove that $A_{z}=\left\{0, q_{n-1}\right\}$. To do this, first notice that clearly $\left\{0, q_{n-1}\right\} \subset A_{z}$. Then, consider any $i \in \mathbb{N}$ with $0<i<q_{n-1}$. Observe that by Lemma 4.1 we have $F^{i}(z) \notin \hat{I}_{n-1}(\hat{x})$. On the other hand, if $F^{i}(z)$ belonged to $\hat{I}_{n-1}\left(F^{q_{n-1}}(\hat{x})\right)$, we would have $\left\{F^{i}(z), F^{q_{n-1}-i}\left(F^{i}(z)\right)\right\} \subset \hat{I}_{n-1}\left(F^{q_{n-1}}(\hat{x})\right)$, which clearly contradicts Lemma 4.1.

Now let $j$ be any natural number with $q_{n-1}<j<q_{n}$. Applying Lemma 4.1 once again we know $F^{j}(z) \notin \hat{I}_{n-1}(z)$. On the other hand, if $F^{j}(z)$ belonged to $\hat{I}_{n-1}\left(F^{q_{n-1}}(\hat{x})\right)$, it would hold $\left\{F^{q_{n-1}}(z), F^{j-q_{n-1}}\left(F^{q_{n-1}}(z)\right)\right\} \subset \hat{I}_{n-1}\left(F^{q_{n-1}}(\hat{x})\right)$, which contradicts Lemma 4.1, too. Thus, $A_{z}=\left\{0, q_{n-1}\right\}$.

Now, by (6.12), it holds

$$
\psi_{\Gamma}^{0,1}(y)=\bar{\phi}_{n}(y)-\xi(y)-\xi\left(f_{n-1}(y)\right)=0,
$$

for every $y \in I_{n-1}\left(x^{\star}\right)=\left[x^{\star}, f_{\Gamma}^{1,0}\left(x^{\star}\right)\right]$.

Finally, putting together (6.26), 6.25) and Proposition 5.3 we conclude $\Gamma$ is a $C^{r}$-coboundary, and by Lemma 5.1, $\tilde{\phi} \in B\left(F, C^{r}(\mathbb{T})\right)$, as desired.

\section{Proof of Theorem A}

First of all, let us suppose $\rho(F)$ is Diophantine. Then, by Herman-Yoccoz theorem [Her79, Yoc84] $F$ is smoothly conjugate to the rigid rotation $R_{\rho(F)}$ and, by Proposition 2.3, we know $\operatorname{dim} \mathcal{D}^{\prime}\left(R_{\rho(F)}\right)=1$. Then, $\mathcal{D}^{\prime}(F)$ is one-dimensional, too, i.e. $\mathcal{D}^{\prime}(F)$ is spanned by the only $F$-invariant probability measure.

Therefore, from now on we can assume $F$ exhibits a Liouville rotation number, and Theorem A will follow as a straightforward consequence of the following result, which can be considered as a finitary version of it: 
Theorem 7.1. Let $F \in \operatorname{Diff}_{+}^{r}(\mathbb{T})$ (with $r \geq 5$ ) be such that $\rho(F)$ satisfies the following condition: the set $\mathcal{L}(\alpha, r / 2)$ given by (2.12) contains infinitely many elements for some, and hence any, $\alpha \in \pi^{-1}(\rho(F)) \subset \mathbb{R}$ (e.g when $\rho(F)$ is Liouville).

Let $k:=\left\lfloor\frac{r-5}{6}\right\rfloor$ and $\phi \in C^{k}(\mathbb{T})$ be such that

$$
\int_{\mathbb{T}} \phi \mathrm{d} \mu=0,
$$

where $\mu$ is the only F-invariant probability measure.

Then, given any $\epsilon>0$, there exists $\tilde{\phi} \in C^{k}(\mathbb{T})$ such that $\tilde{\phi} \in B\left(F, C^{k}(\mathbb{T})\right)$ and

$$
\|\tilde{\phi}-\phi\|_{C^{k}} \leq \epsilon .
$$

Notice that, by Proposition 2.2 the conclusion of this theorem can be briefly summarized saying that $\mathcal{D}_{k}^{\prime}(F)=\mathbb{R} \mu$.

Proof of Theorem 7.1. First, let us fix a lift $f \in \widetilde{\operatorname{Diff}_{+}^{r}}(\mathbb{T})$ of $F$ and then we can suppose $\alpha:=\rho(f)$. Let $\left(q_{n}\right)$ and $\left(\beta_{n}\right)$ the sequences given by (2.5) and (2.8) associated to the continued fraction expansion of $\alpha$.

By our arithmetical hypothesis $\mathcal{L}(\alpha, r / 2)$ is a infinite set and so, we can find $n \in$ $\mathcal{L}(\alpha, r / 2)$ with $n \geq n_{0}$, where $n_{0}$ is the natural number given by Proposition 4.7 Let $x^{\star} \in \mathbb{R}$ be any point such that $m_{n-1}\left(x^{\star}\right)=M_{n-1}$.

Now, by combining Proposition 4.6 and Proposition 4.7, for any $0 \leq s \leq r-2$ and any $y \in K_{n-1}\left(x^{\star}\right)$ we have

$$
\begin{aligned}
\mid D^{s} \log D & f_{n}(y) \mid \leq C \frac{\sqrt{M_{n-1}}}{\left(m_{n-1}\left(x^{\star}\right)\right)^{s}}\left(\left(\sqrt{M_{n-1}}\right)^{r-2}+\frac{m_{n}\left(x^{\star}\right)}{m_{n-1}\left(x^{\star}\right)}\right) \\
\leq & C\left(\left(M_{n-1}\right)^{\frac{r-1}{2}-s}+\frac{\beta_{n}}{\beta_{n-1}}\left(M_{n-1}\right)^{\frac{1}{2}-s}\right) \\
\leq & C\left(\left(M_{n-1}\right)^{\frac{r-1}{2}-s}+\beta_{n-1}^{\frac{r}{2}-1}\left(M_{n-1}\right)^{\frac{1}{2}-s}\right) \\
& \leq C\left(M_{n-1}\right)^{\frac{r-1}{2}-s},
\end{aligned}
$$

where the last inequality is consequence of the fact that

$$
\beta_{n-1}=\int_{\mathbb{T}} m_{n-1}(t) \mathrm{d} \mu(t) \leq M_{n-1} .
$$

Then, if $P_{s} \in \mathbb{Z}\left[X_{1}, \ldots, X_{s}\right]$ denotes the polynomial of degree $s$ given by Proposition 4.8, applying (4.7) and (7.2) we get

$$
\begin{aligned}
\left|D^{s+1} f_{n}(y)\right| & =\left|P_{s}\left(D \log D f_{n}(y), \ldots, D^{s} \log D f_{n}(y)\right) D f_{n}(y)\right| \\
& \leq C P_{s}\left(\left(M_{n-1}\right)^{\frac{r-1}{2}-1},\left(M_{n-1}\right)^{\frac{r-1}{2}-2}, \ldots,\left(M_{n-1}\right)^{\frac{r-1}{2}-s}\right) \\
& \leq C\left(\frac{1}{M_{n-1}}\right)^{s} P_{s}\left(\left(M_{n-1}\right)^{\frac{r-1}{2}}, \ldots,\left(M_{n-1}\right)^{\frac{r-1}{2}}\right) \\
& \leq C\left(M_{n-1}\right)^{s \frac{r-1}{2}-s}=C\left(\sqrt{M_{n-1}}\right)^{r s-3 s},
\end{aligned}
$$


for every $y \in K_{n-1}\left(x^{\star}\right)$.

Now, let $u \in C^{r}(\mathbb{T})$ be the function we constructed in Lemma 6.1 We affirm that we can find a constant $C>0$, depending only on $f$ and $r$, such that

$$
\left\|u f_{n}-\left.u\right|_{I_{n-1}\left(x^{\star}\right)}\right\|_{C^{s}} \leq C\|\phi\|_{C^{s+1}}\left(\sqrt{M_{n-1}}\right)^{r-3 s-4}
$$

To prove this, first notice that for any $0 \leq s \leq r-2$ and $y \in I_{n-1}\left(x^{\star}\right)$, we have

$$
\begin{aligned}
& \left|D^{s} u\left(f_{n}(y)\right)-D^{s} u(y)\right| \leq \int_{y}^{f_{n}(y)}\left|D^{s+1} u(t)\right| \mathrm{d} \operatorname{Leb}(t) \\
& \quad \leq m_{n}(y)\left\|\left.D^{s+1} u\right|_{I_{n-1}\left(x^{\star}\right)}\right\|_{C^{0}} \\
& \quad \leq C \frac{\beta_{n}}{\beta_{n-1}} M_{n-1}\left\|\left.\phi_{n-1}\right|_{K_{n-1}\left(x^{\star}\right)}\right\|_{C^{s+1}} \Theta(\alpha, n, s+1)\left(\frac{1}{M_{n-1}}\right)^{s+1} \\
& \quad \leq C \beta_{n-1}^{\frac{r}{2}-1}\|\phi\|_{C^{s+1}}\left(\frac{1}{\sqrt{M_{n-1}}}\right)^{s+2}\left(\frac{1}{1-\beta_{n-1}^{r / 2-1}}\right)^{s+1}\left(\frac{1}{M_{n-1}}\right)^{s} \\
& \quad \leq C\|\phi\|_{C^{s+1}}\left(M_{n-1}\right)^{\frac{r}{2}-1}\left(\frac{1}{M_{n-1}}\right)^{\frac{3 s+2}{2}}=C\|\phi\|_{C^{s+1}}\left(\sqrt{M_{n-1}}\right)^{r-3 s-4} .
\end{aligned}
$$

Observe that (7.5) is indeed the proof of (7.4) for the particular case $s=0$.

In the other cases, that is when $s \geq 1$, we can use Faa-di Bruno equation and estimates (7.2), (7.3) and (7.5) to prove (7.4):

$$
\begin{aligned}
& \left|D^{s}\left(u f_{n}-u\right)(y)\right| \leq\left|D^{s} u\left(f_{n}(y)\right) D f_{n}(y)-D^{s} u(y)\right| \\
& +\left|\sum_{j=1}^{s-1} D^{j} u\left(f_{n}(y)\right) B_{s, j}\left(D f_{n}(y), \ldots, D^{s-j+1} f_{n}(y)\right)\right| \\
& \leq D f_{n}(y)\left|D^{s} u\left(f_{n}(y)\right)-D^{s} u(y)\right|+\left|D f_{n}(y)-1\right|\left|D^{s} u(y)\right| \\
& +C \sum_{j=1}^{s-1}\left\|\left.\phi_{n-1}\right|_{K_{n-1}\left(x^{\star}\right)}\right\|_{C^{j}} \Theta(\alpha, n, j)\left(\frac{1}{M_{n-1}}\right)^{j} \\
& B_{s, j}\left(1,\left(\sqrt{M_{n-1}}\right)^{r-3}, \ldots,\left(\sqrt{M_{n-1}}\right)^{(r-3)(s-j)}\right) \\
& \leq C\left(\|\phi\|_{C^{s+1}} M_{n-1}^{\frac{r-3 s-4}{2}}+M_{n-1}^{\frac{r-1}{2}}\left\|\left.\phi_{n-1}\right|_{K_{n-1}\left(x^{\star}\right)}\right\|_{C^{s}} M_{n-1}^{-s}\right) \\
& +C \sum_{j=1}^{s-1}\|\phi\|_{C^{j}}\left(\frac{1}{\sqrt{M_{n-1}}}\right)^{j+1}\left(\frac{1}{M_{n-1}}\right)^{j}\left(\sqrt{M_{n-1}}\right)^{(r-3)(s-j)} \\
& \leq C\|\phi\|_{C^{s+1}}\left[\left(\sqrt{M_{n-1}}\right)^{r-3 s-4}+\left(\sqrt{M_{n-1}}\right)^{r-3 s-1}\right] \\
& \leq C\|\phi\|_{C^{s+1}}\left(\sqrt{M_{n-1}}\right)^{r-3 s-4} \text {, }
\end{aligned}
$$


and (7.4) is proved.

Now, let us consider the cocycle $\bar{\phi}$ as defined in Lemma 6.1, i.e. given by $\bar{\phi}:=$ $\phi+u-u f$. Notice that whenever $0 \leq s \leq \frac{2 r-3}{3}$, combing Proposition 4.12 and (7.4) we get

$$
\left|D^{s} \bar{\phi}_{n}(y)\right| \leq\left|D^{s} \phi_{n}(y)\right|+\left|D^{s}\left(u-u f_{n}\right)(y)\right| \leq C\|\phi\|_{C^{s+1}}\left(\frac{1}{\sqrt{M_{n-1}}}\right)^{s+1},
$$

for every $y \in I_{n-1}\left(x^{\star}\right)$.

On the other hand, remember that in the middle of the proof of Lemma 6.2 we show that $\bar{\phi}$ satisfies (6.18), that is

$$
\bar{\phi}_{n}(y)=\bar{\phi}_{n}\left(f_{n-1}(y)\right), \quad \forall y \in I_{n-1}\left(x^{\star}\right) .
$$

This implies there exists a unique function $\gamma \in C^{r}(\mathbb{R})$ which coincides with $\bar{\phi}_{n}$ on $I_{n-1}\left(x^{\star}\right)$ and is $f_{n-1}$-invariant on the whole real line. To estimate the $C^{r}$-norm of $\gamma$ first observe that, by the definition of $\gamma$ and estimate (7.7), it holds

$$
\left\|\left.\gamma\right|_{I_{n-1}\left(x^{\star}\right)}\right\|_{C^{s}}=\left\|\left.\bar{\phi}_{n}\right|_{I_{n-1}\left(x^{\star}\right)}\right\|_{C^{s}} \leq C\|\phi\|_{C^{s+1}}\left(\frac{1}{\sqrt{M_{n-1}}}\right)^{s+1} .
$$

On the other hand, applying estimate (7.7) and recalling $\gamma=\gamma \circ f_{n-1}$, for any $y \in I_{n-1}\left(f_{n-1}\left(x^{\star}\right)\right)$ we get

$$
\begin{aligned}
\left|D^{s} \gamma(y)\right| & =\left|D^{s}\left(\gamma \circ f_{n-1}^{-1}\right)(y)\right|=\left|D^{s}\left(\bar{\phi}_{n} \circ f_{n-1}^{-1}\right)(y)\right| \\
& =\left|\sum_{i=1}^{s} D^{i} \bar{\phi}_{n}\left(f_{n-1}^{-1}(y)\right) B_{s, i}\left(D f_{n-1}^{-1}(y), \ldots, D^{s-i+1} f_{n-1}^{-1}(y)\right)\right| \\
& \leq\left.\sum_{i=1}^{s}\left\|\left.\bar{\phi}\right|_{I_{n-1}\left(x^{\star}\right)}\right\|\right|_{C^{i}}\left|B_{s, i}\left(D f_{n-1}^{-1}(y), \ldots, D^{s-i+1} f_{n-1}^{-1}(y)\right)\right| \\
& \leq C\|\phi\|_{C^{s+1}} \sum_{i=1}^{s}\left(\frac{1}{\sqrt{M_{n-1}}}\right)^{i+1}\left(D f_{n-1}^{-1}(y)\right)^{i}\left(\frac{1}{\sqrt{M_{n-1}}}\right)^{s-i} \\
& =C\|\phi\|_{C^{s+1}}\left(\frac{1}{\sqrt{M_{n-1}}}\right)^{s+1} .
\end{aligned}
$$

Repeating this argument we can show that, given any $k \in \mathbb{N}$, there exists a constant $C_{k}>0$ depending only on $f, r$ and $k$ such that

$$
\left|D^{s} \gamma(y)\right| \leq C_{k}\|\phi\|_{C^{s+1}}\left(\frac{1}{\sqrt{M_{n-1}}}\right)^{s+1}, \quad \forall y \in I_{n-1}\left(f_{n-1}^{k}\left(x^{\star}\right)\right) .
$$

Now, returning to (7.8) we can affirm there exists $x_{1} \in I_{n-1}\left(x^{\star}\right)$ satisfying

$$
D \gamma\left(x_{1}\right)=D \bar{\phi}_{n}\left(x_{1}\right)=0 .
$$


Moreover, since $\gamma$ is $f_{n-1}$-periodic and $f_{n-1}$ is a diffeomorphism, $x_{1}^{k}:=f_{n-1}^{k}\left(x_{1}\right)$ is a critical point of $\gamma$, for every $k \in \mathbb{Z}$.

This implies that, for each integer $k$, we can find a point $x_{2}^{k} \in\left[f_{n-1}^{k}\left(x_{1}\right), f_{n-1}^{k+1}\left(x_{1}\right)\right]$ such that $D^{2} \gamma\left(x_{2}^{k}\right)=0$, and inductively, we can define the sequence (of sequences) of points $\left(x_{s}^{k}\right)_{1 \leq s \leq r, k \in \mathbb{Z}}$ that satisfies

$$
D^{s} \gamma\left(x_{s}^{k}\right)=0, \quad \text { and } \quad x_{s+1}^{k} \in\left[x_{s}^{k}, x_{s}^{k+1}\right] \subset \mathbb{R},
$$

for every $1 \leq s \leq r$ and every $k \in \mathbb{Z}$. Now, if we write

$$
\mathcal{I}_{s}:=I_{n-1}\left(x^{\star}\right) \cup I_{n-1}\left(f_{n-1}\left(x^{\star}\right)\right) \cup \cdots \cup I_{n-1}\left(f_{n-1}^{s-1}\left(x^{\star}\right)\right),
$$

one can easily check that

$$
x_{s}^{0} \in \mathcal{I}_{s} \quad \text { and } \quad \operatorname{Leb}\left(\mathcal{I}_{s}\right) \leq s M_{n-1} .
$$

Now, estimates (7.10), (7.11) and (7.12) can be used to improved our estimate (7.7). In fact, if $0 \leq s \leq \frac{2 r-3}{3}$, one can easily check that

$$
\begin{aligned}
\left|D^{s-1} \gamma(y)\right| & \leq \int_{x_{s}^{0}}^{y}\left|D^{s} \gamma(z) \mathrm{d} z\right| \leq C\|\phi\|_{C^{s+1}}\left(\frac{1}{\sqrt{M_{n-1}}}\right)^{s+1} s M_{n-1} \\
& =C\|\phi\|_{C^{s+1}} M_{n-1}^{1-\frac{s+1}{2}}, \quad \forall y \in \mathcal{I}_{s} .
\end{aligned}
$$

Iterating this procedure of integration from the appropriate point $x_{s}^{0}$ we get

$$
\begin{aligned}
\left|D^{\bar{s}-j} \bar{\phi}_{n}(y)\right| & =\left|D^{\bar{s}-j} \gamma(y)\right| \leq C\|\phi\|_{C^{\bar{s}+1}}\left(\frac{1}{\sqrt{M_{n-1}}}\right)^{\bar{s}+1} M_{n-1}^{j} \\
& =C\|\phi\|_{C^{\bar{s}+1}} M_{n-1}^{j-\frac{\bar{s}+1}{2}}, \quad \forall y \in I_{n-1}\left(x^{\star}\right),
\end{aligned}
$$

and each $j \in\{0,1, \ldots, \bar{s}-1\}$, where $\bar{s}:=\left\lfloor\frac{2 r-3}{3}\right\rfloor$.

Now recall we are assuming $\phi$ has zero average with respect to $\mu$. Since $\bar{\phi}$ is is cohomologous to $\phi$, the same holds for $\bar{\phi}$. Therefore, there must exist a point $x_{0} \in$ $I_{n-1}\left(x^{\star}\right)$ such that $\bar{\phi}_{n}\left(x_{0}\right)=0$. In particular, estimate (7.14) also holds for $j=\bar{s}$. Then, recalling the number $k$ is equal to $\left\lfloor\frac{r-5}{6}\right\rfloor$, we have

$$
\left\|\left.\bar{\phi}_{n}\right|_{I_{n-1}\left(x^{\star}\right)}\right\|_{C^{k}} \leq C\|\phi\|_{C^{\bar{s}+1}} M_{n-1}^{\bar{s}-k-\frac{\bar{s}+1}{2}} \leq C\|\phi\|_{C^{r}} M_{n-1}^{\frac{r-3}{6}} .
$$

Then, we apply Lemma 6.2 to construct the function $\xi \in C^{r}(\mathbb{T})$ and putting together estimates (6.13) and (7.15) we obtain

$$
\|\xi\|_{C^{k}} \leq C M_{n-1}^{-k}\left\|\left.\bar{\phi}_{n}\right|_{I_{n-1}\left(x^{\star}\right)}\right\|_{C^{k}} \leq C\|\phi\|_{C^{r}} M_{n-1}^{\frac{r-3}{6}-k} \leq C\|\phi\|_{C^{r}} M_{n-1}^{\frac{1}{3}}
$$

Taking into account that $C$ is a real constant which only depends on $F$ and $r$, that, by the minimality, $M_{m} \rightarrow 0$ as $m \rightarrow+\infty$ and that $\mathcal{L}(\alpha, r / 2)$ has infinitely many elements, we conclude we can choose $n \in \mathcal{L}(\alpha, r / 2)$ big enough such that $C\|\phi\|_{C^{r}} M_{n-1}^{1 / 3} \leq \epsilon$.

Finally, by Lemma 6.3 the cocycle $\tilde{\phi}:=\phi-\xi$ is a $C^{r}$-coboundary for $F$, and by the previous remark we have $\|\tilde{\phi}-\phi\|_{C^{k}}=\|\xi\|_{C^{k}}<\epsilon$, as desired. 


\section{References}

[AK09] A. Avila and A. Kocsard, Invariant distributions for higher dimensional quasiperiodic maps, in preparation, 2009.

[dMvS93] W. de Melo and S. van Strien, One-dimensional dynamics, Ergebnisse der Mathematik und ihrer Grenzgebiete (3) [Results in Mathematics and Related Areas (3)], vol. 25, Springer-Verlag, Berlin, 1993. MR MR1239171 (95a:58035)

[Ghy89] Étienne Ghys, L'invariant de Godbillon-Vey, Astérisque (1989), no. 177-178, 155-181, Séminaire Bourbaki, Vol. 1988/89. MR 1040572 (91h:57015)

[Ghy01] Groups acting on the circle, Enseign. Math. (2) 47 (2001), no. 3-4, 329-407. MR MR1876932 (2003a:37032)

[Ghy08] É. Ghys, Groups acting on the circle: a selection of open problems, talk given during the IIIéme Cycle Romand de Mathématiques at Les Diablerets. Slides available in www.unige.ch/ ${ }^{\sim}$ tatiana/Diablerets08/ghys_diablerets.pdf, March 2008.

[HB83] A. Haefliger and L. Banghe, Currents on a circle invariant by a Fuchsian group, Geometric dynamics (Rio de Janeiro, 1981), Lecture Notes in Math., vol. 1007, Springer, Berlin, 1983, pp. 369-378. MR MR730277 (85k:57028)

[Her79] M. Herman, Sur la conjugaison différentiable des difféomorphismes du cercle à des rotations, Inst. Hautes Études Sci. Publ. Math. (1979), no. 49, 5-233.

[Hur02] Steven Hurder, Dynamics and the Godbillon-Vey class: a history and survey, Foliations: geometry and dynamics (Warsaw, 2000), World Sci. Publ., River Edge, NJ, 2002, pp. 29-60. MR 1882764 (2003b:37044)

[HW79] G. H. Hardy and E. M. Wright, An introduction to the theory of numbers, fifth ed., The Clarendon Press Oxford University Press, New York, 1979. MR MR568909 (81i:10002)

[Kat01] A. Katok, Cocycles, cohomology and combinatorial constructions in ergodic theory, Smooth ergodic theory and its applications (Seattle, WA, 1999) (Providence, RI), Proc. Sympos. Pure Math., vol. 69, Amer. Math. Soc., 2001, In collaboration with E. A. Robinson, Jr., pp. 107-173.

[Kat03] - Combinatorial constructions in ergodic theory and dynamics, University Lecture Series, vol. 30, American Mathematical Society, Providence, RI, 2003. MR MR2008435 (2004m:37005)

[Lan94] R. Langevin, A list of questions about foliations, Differential topology, foliations, and group actions (Rio de Janeiro, 1992), Contemp. Math., vol. 161, Amer. Math. Soc., Providence, RI, 1994, pp. 59-80. MR MR1271828 (94m:57057) 
[Nav02] Andrés Navas, Actions de groupes de Kazhdan sur le cercle, Ann. Sci. École Norm. Sup. (4) 35 (2002), no. 5, 749-758. MR 1951442 (2003j:58013)

[Yoc84] J.-C. Yoccoz, Conjugaison différentiable des difféomorphismes du cercle dont le nombre de rotation vérifie une condition diophantienne, Ann. Sci. École Norm. Sup. (4) 17 (1984), no. 3, 333-359.

[Yoc95] - Centralisateurs et conjugaison différentiable des difféomorphismes du cercle, Astérisque (1995), no. 231, 89-242, Petits diviseurs en dimension 1 .

CNRS 7586, Institut de Mathématiques de Jussieu, 175 Rue du Chevaleret, 75013, PARIS - France.

E-mail addres: artur@math. sunysb.edu

Instituto de Matemática, Universidade Federal Fluminense. Rua Mário Santo Braga, s/n. Niterói, RJ - Brazil

E-mail address: alejandro@mat.uff.br 